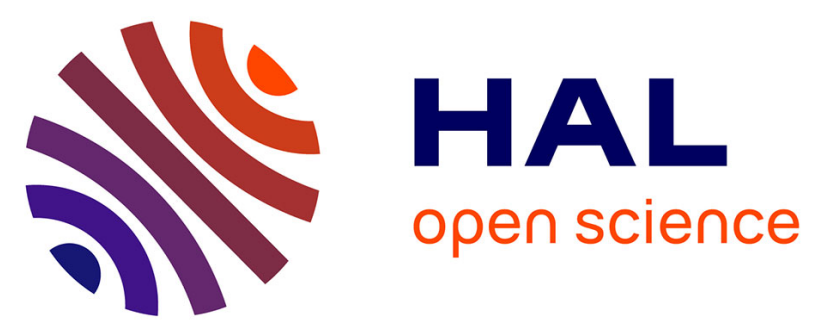

\title{
CFD modelling of particle resuspension in a toroidal geometry resulting from airflows during a loss of vacuum accident (LOVA)
}

Thomas Gelain, Francois Gensdarmes, Samuel Peillon, Laurent Ricciardi

\section{- To cite this version:}

Thomas Gelain, Francois Gensdarmes, Samuel Peillon, Laurent Ricciardi. CFD modelling of particle resuspension in a toroidal geometry resulting from airflows during a loss of vacuum accident (LOVA). Fusion Engineering and Design, 2020, 151, pp.111386. 10.1016/j.fusengdes.2019.111386 . hal-02444483

\section{HAL Id: hal-02444483 \\ https://hal.science/hal-02444483}

Submitted on 17 Jan 2020

HAL is a multi-disciplinary open access archive for the deposit and dissemination of scientific research documents, whether they are published or not. The documents may come from teaching and research institutions in France or abroad, or from public or private research centers.
L'archive ouverte pluridisciplinaire HAL, est destinée au dépôt et à la diffusion de documents scientifiques de niveau recherche, publiés ou non, émanant des établissements d'enseignement et de recherche français ou étrangers, des laboratoires publics ou privés.

\section{(1) (1) $\$$}

Distributed under a Creative Commons Attribution - NonCommercial - NoDerivatives $\mid 4.0$ 


\title{
CFD modelling of particle resuspension in a toroidal geometry resulting from airflows during a loss of vacuum accident (LOVA)
}

Thomas Gelain, François Gensdarmes, Samuel Peillon, Laurent Ricciardi Institut de Radioprotection et de Sûreté Nucléaire (IRSN), PSN-RES, SCA, Gif-sur-Yvette 91192, France

\begin{abstract}
:
During a loss of vacuum accident (LOVA), dusts that will be present in the future tokamak ITER are likely to be resuspended. Such dusts may present a risk of explosion and airborne contamination. Hence, an assessment of the amount of particles which may be resuspended in case of LOVA is a major safety issue in ITER and more widely for all the fusion reactors. This article presents the implementation of the Rock'n'Roll model for the particle resuspension in ANSYS CFX and its coupling with a validated particles transport and deposition model.
\end{abstract}

After reminding the main phenomena contributing to the particle resuspension and their equations, this article proposes in a first time a checking of the good implementation of the resuspension model in ANSYS CFX. To do that, numerical results are compared to analytical calculations and experimental results acquired on facilities dedicated to the study of the resuspension.

After this first step, this article proposes an application for a LOVA scenario in a toroidal vessel in order to demonstrate the feasibility of this kind of calculation in conditions similar to those encountered in a tokamak type geometry and the robustness of the coupling between the particle transport model and the resuspension model.

The final application for modelling a LOVA in a toroidal geometry with different particle sizes shows a good behaviour of the coupled resuspension and transport models and a good agreement of the results with the expected physics.

However, it must be reminded that this numerical application is a first step towards the analysis of dust resuspension and transport in a tokamak like ITER and the interpretation of quantitative results presented here should remain in this specific context. 


\section{INTRODUCTION}

As part of the ITER construction, it is important to develop knowledge that ensures the highest level of safety in the nuclear facility [1]. To assess the safety of nuclear facilities and the relevance of associated radiation protection measures, a key step consists in determining source terms of contamination during normal operation and for various accident scenarii. Since it concerns potential contamination in aerosol form, source terms are calculated using resuspension coefficients which represent the amount of resuspended aerosol compared to the amount of matter that may be potentially dispersed, depending on the scenario.

In ITER's normal operating conditions, several hundred kilogrammes of particles made of beryllium (Be) and tungsten (W) will be produced following the erosion of the vacuum vessel (VV) walls (blanket and divertor) by the plasma [2]. The particles are subject to the conditions found in the VV and may thus be radioactive (tritium and activation products [3], exhibit acute chemical toxicity (beryllium particles) [4] [5], or constitute, with air or water vapour, a potentially explosive mixture [2].

The mobility of these particles and their containment in the event of a LOVA have become, over time, a major topic for assessing the safety of ITER [6] [7] [8] [9] [10]

Several studies have been conducted in order to understand the phenomenon of particle resuspension that highlight the main driver which is the friction velocity [11] [12] [13]. These studies also helped to characterize the competition between the airflow forces and the adhesive forces, for different particle diameters and to develop the resuspension Rock'n'Roll model [14] $[15]$.

Considering this latter, its application can be enforced in order to evaluate the risks caused by the particle resuspension in case of LOVA. To do this, the Rock'n'Roll model was implemented in a CFD code and precisely compared to experimental data.

This work develops the implementation of the Rock'n'Roll model in a CFD code (ANSYS CFX) and presents the different steps of its validation with experimental data acquired on IRSN facilities, before detailing its application to the case of a LOVA in a toroidal geometry.

Although the application proposed is related to the specific issue of a LOVA, the hazards related to the particle resuspension are relevant to numerous industrial situations in which powders are manipulated, can fall on the floor and be resuspended by operator displacements. Hence, the present calculations could be extended to wider industrial applications. 


\section{OVERVIEW OF PARTICLES RESUSPENSION BY AN AIRFLOW}

Particles resuspension consists in detaching particles from the location where they are deposited by an external force, generally an airflow, and entraining them. The phenomenon involves two forces, attractive and entrainment. Attractive forces tend to maintain the particles on the wall and consist essentially of gravity, capillarity and Van der Walls adhesive forces that are described by the following equations:

- gravity force $\mathrm{F}_{\mathrm{G}}$ :

$$
\mathrm{F}_{\mathrm{G}}=\frac{\pi}{6} \rho_{p} d_{p}^{3} g
$$

Where $\rho_{p}$ is the particle density $\left(\mathrm{kg} \cdot \mathrm{m}^{-3}\right), d_{p}$ is the particle diameter $(\mathrm{m})$ and $g$ is the gravity $\left(\mathrm{m} \cdot \mathrm{s}^{-2}\right)$.

- capillarity force $\mathrm{F}_{\mathrm{C}}$ :

$$
\mathrm{F}_{\mathrm{C}}=2 \pi \sigma d_{p}
$$

Where $\sigma$ is the water surface tension $\left(\mathrm{N} \cdot \mathrm{m}^{-1}\right)$.

- Adhesive (Van der Waals) forces $\mathrm{F}_{\mathrm{JKR}}$, also called JKR model:

$$
\mathrm{F}_{\mathrm{JKR}}=\frac{3}{4} \pi \gamma d_{p}
$$

Where $\gamma$ is the surface energy of adhesion $\left(\mathrm{N} \cdot \mathrm{m}^{-1}\right)$ that can be expressed by:

$$
\gamma=\frac{A}{24 \pi z^{2}}
$$

Where $\mathrm{A}$ is the Hamaker constant and $\mathrm{z}$ is the minimal particle-surface distance generally considered equal to $4.10^{-10} \mathrm{~m}[16]$.

Contrary to the attractive forces, the entrainment forces tend to tear the particle off the wall. These forces likely to entrain the particles deposited on a wall are the airflow forces that include the lift and drag forces described by the following equations:

- lift force $\mathrm{F}_{\mathrm{L}}$ :

$$
\mathrm{F}_{\mathrm{L}}=4.2 \rho v^{2}\left(\mathrm{~d}_{\mathrm{p}}^{+}\right)^{2.31}
$$


where $\rho$ is the fluid density $\left(\mathrm{kg} \cdot \mathrm{m}^{-3}\right), \quad v$ is the kinematic viscosity $\left(\mathrm{m}^{2} \cdot \mathrm{s}^{-1}\right)$ and $\mathrm{d}_{\mathrm{p}}^{+}$is the dimensionless particle diameter (-) expressed by:

$$
\mathrm{d}_{\mathrm{p}}^{+}=\frac{d_{p} u^{*}}{v}
$$

where $u^{*}$ is the shear velocity $\left(\mathrm{m} \cdot \mathrm{s}^{-1}\right)$.

- drag force $F_{D}$ :

$$
\mathrm{F}_{\mathrm{D}}=8.01 \rho v^{2}\left(\mathrm{~d}_{\mathrm{p}}^{+}\right)^{2}
$$

The scheme in Figure 1 presents the balance of the forces acting on a particle deposited on a wall

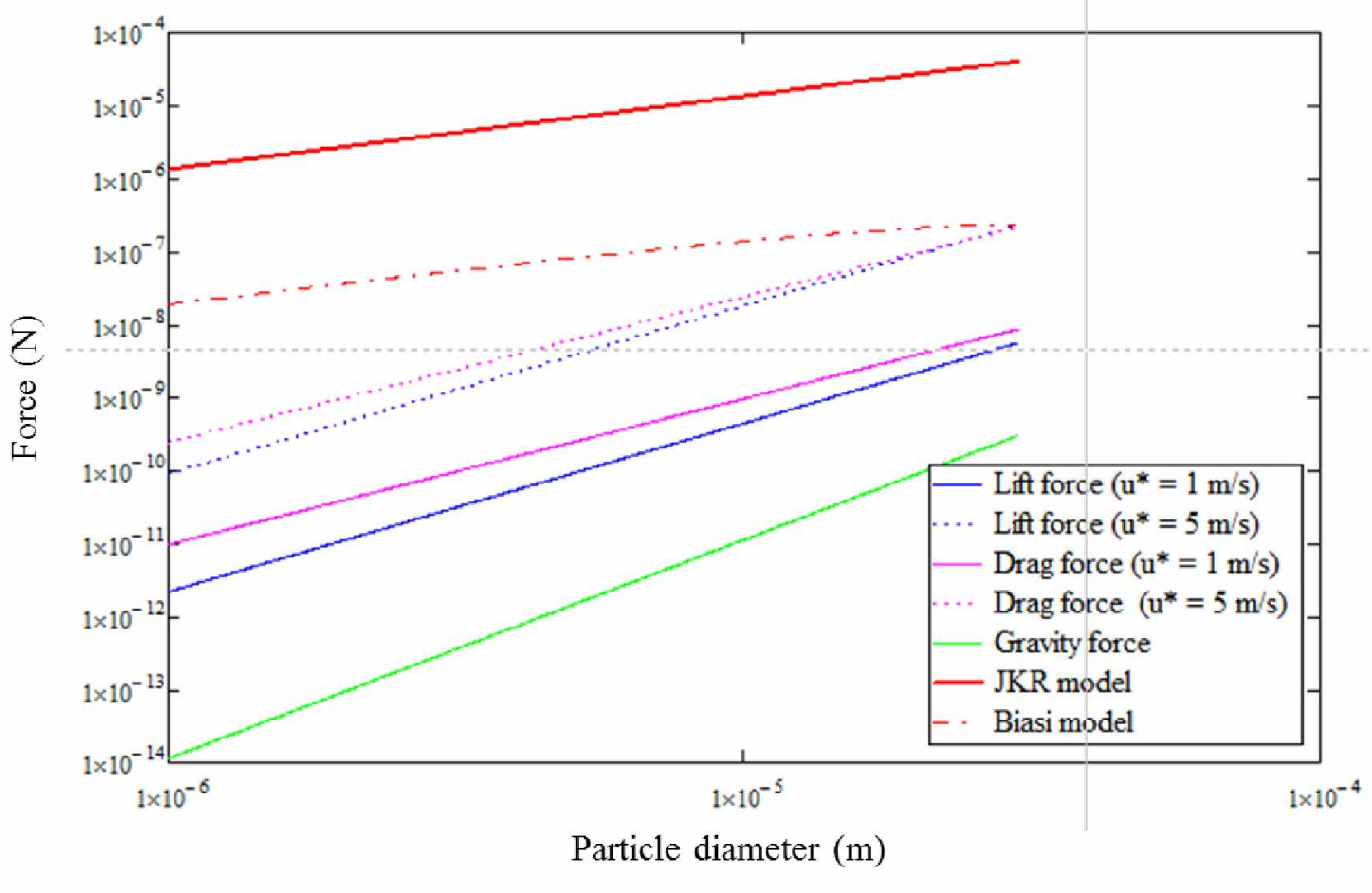

Figure 2 shows the contribution of each of them. 


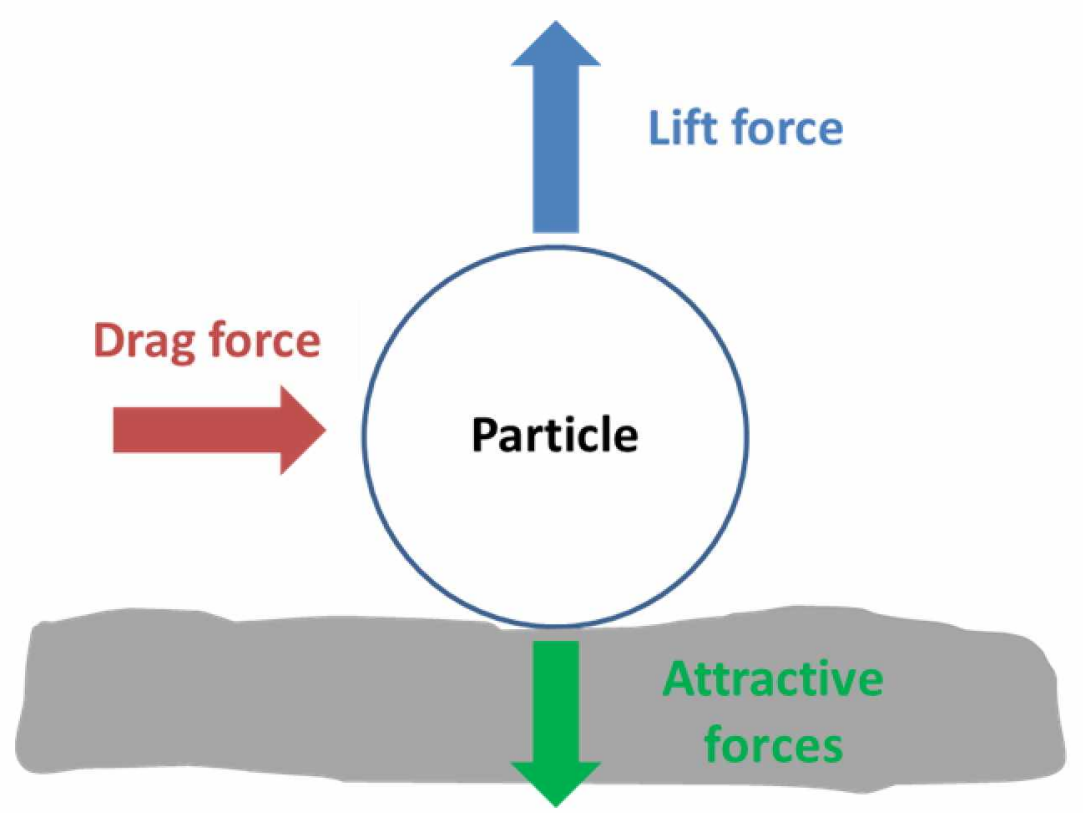

Figure 1 - scheme of forces acting on a particle deposited on a wall

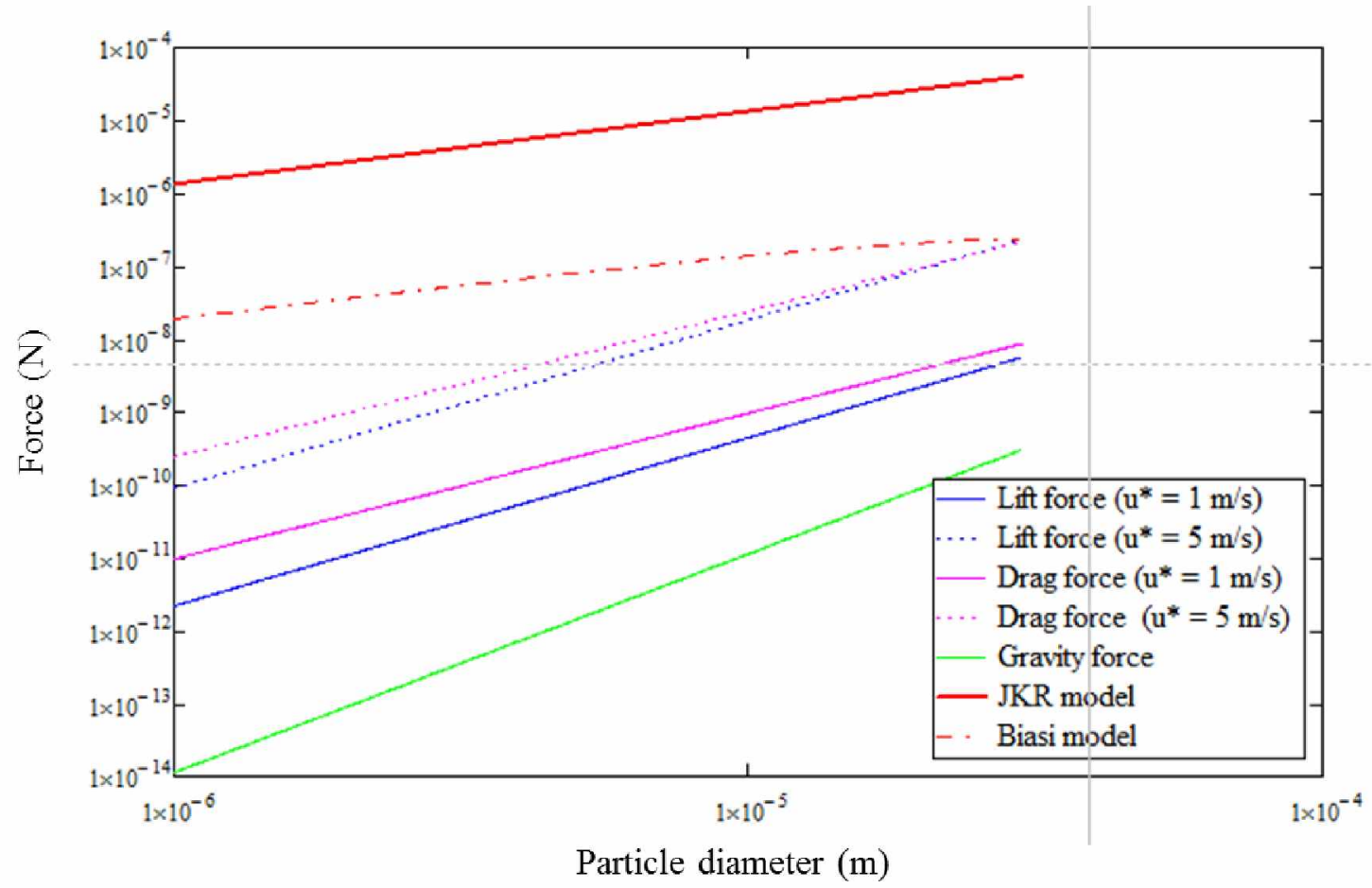

Figure 2 - contribution of the forces acting on a particle deposited on a wall for two values of friction velocity 


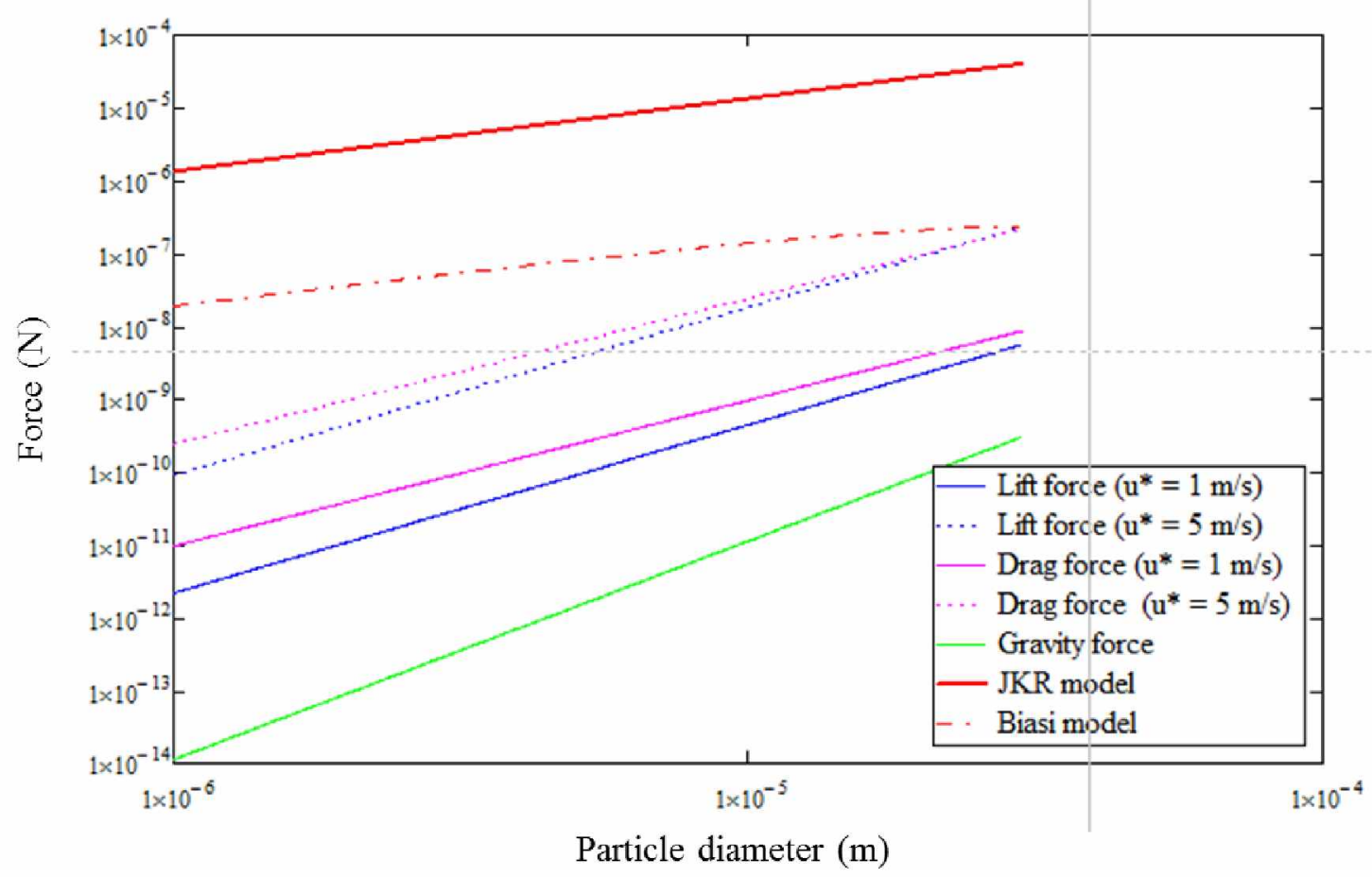

Figure 2 highlights the importance of the adhesion forces compared to the gravity one, regardless the shear velocity or the particle diameter.

For a particle to be resuspended, airflow forces have to be at least equal to the adhesion forces.

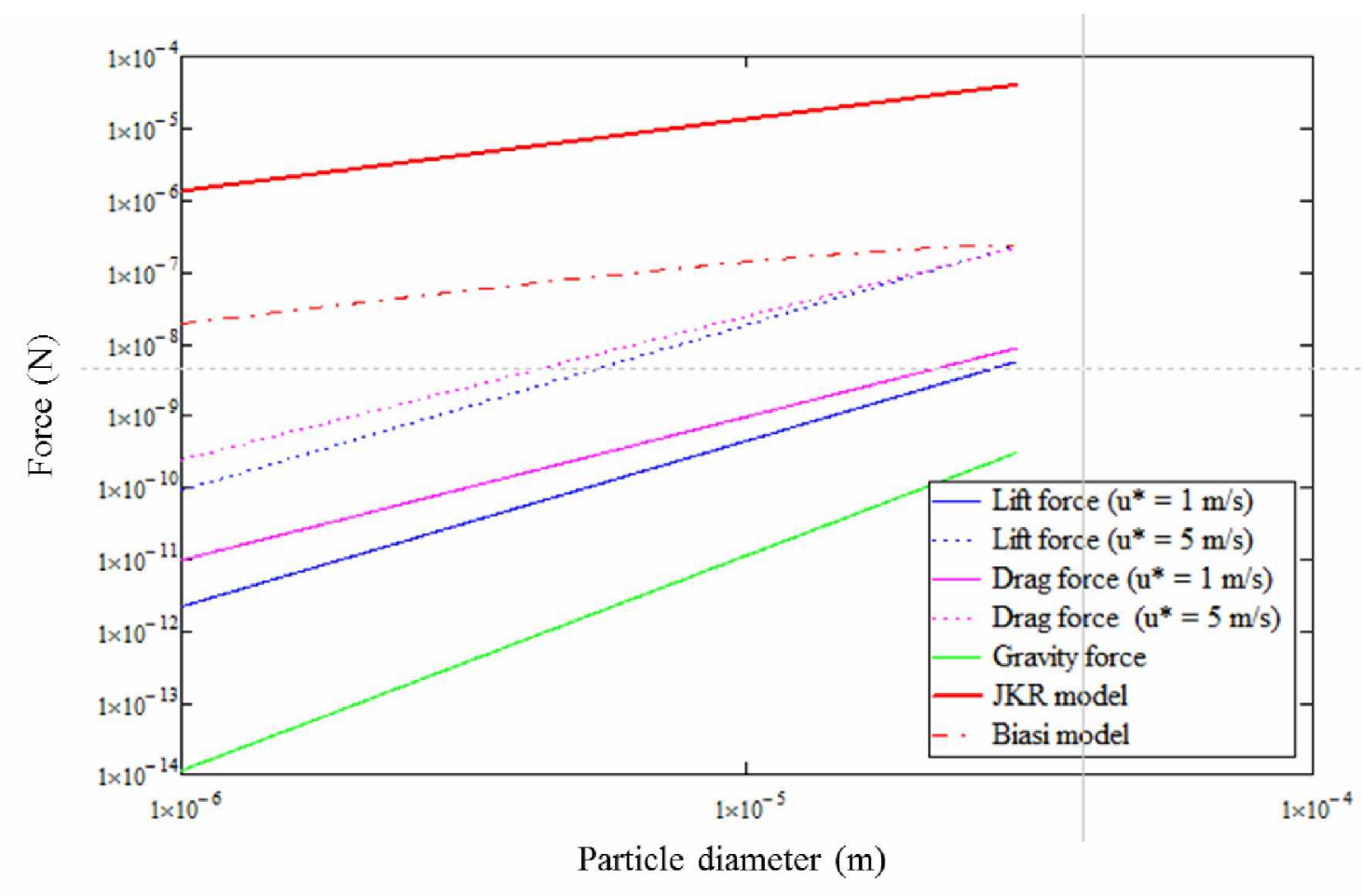

However,

Figure 2 shows that this condition is not reached in the range of the considered particle diameter. The reason is that the adhesion forces (JKR model) are calculated for spherical smooth particles and a smooth wall which are ideal, but not realistic conditions for the adhesion. 
In reality, the particles are not spherical and nor smooth and they are generally deposited on rough surfaces [17]. Hence, adhesion forces cannot be directly calculated by the expression in equation (3). To account for the diminution of adhesion force $F_{J K R}$, due to surface roughness, Biasi et al. [15] tried to determine "universal" correlations for calculating the distribution of adhesion forces. Biasi correlation allows a best fit of Rock'n'Roll model onto many resuspension experiments. It is described in equations (13) and (14) and plotted in

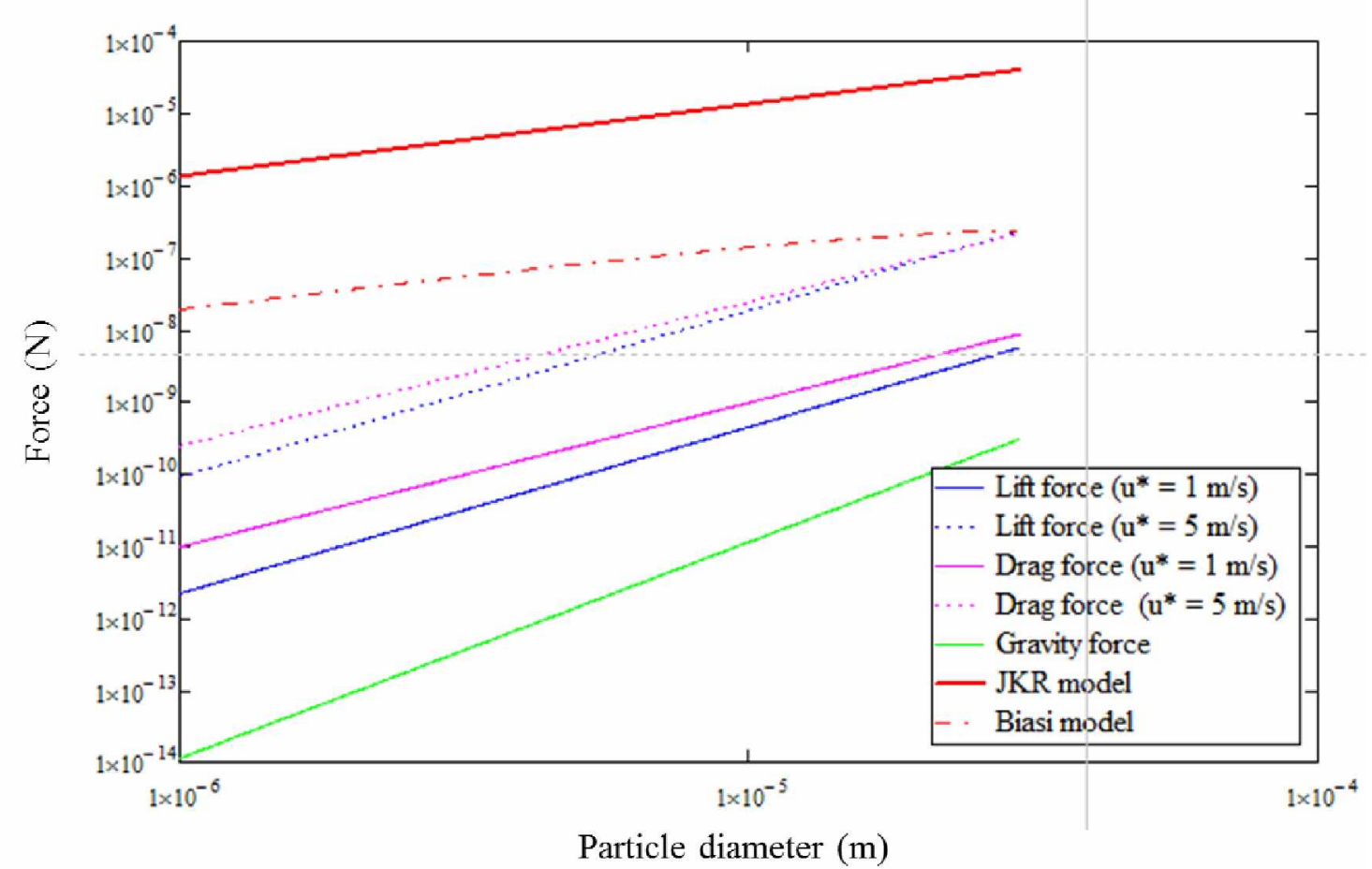

Figure 2 (Biasi model). These correlations may be used in the "Rock'n'Roll" model developed by Reeks \& Hall [14] for a practical particle resuspension calculation.

\section{DESCRIPTION OF THE ROCK'N' ROLL MODEL}

The "Rock'n'Roll" model, developed by Reeks \& Hall [14], on the basis of a so-called "RRH" model developed by Reeks et al. [18], is based on the assumption of a particle deposited on a rough surface which is resuspended by the airflow turbulence effect as shown in Figure 3.
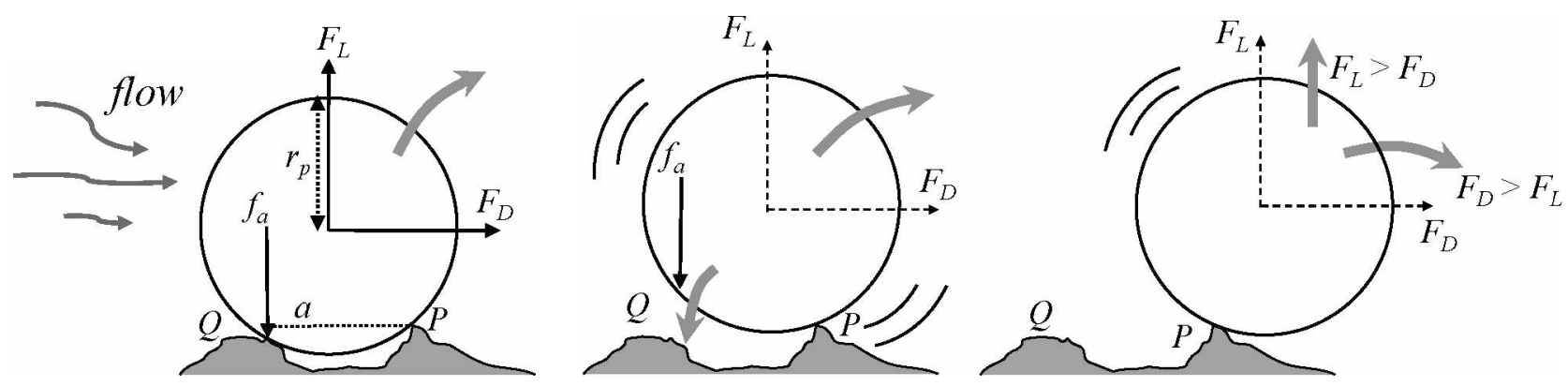
Figure 3 - schematic representation of the "Rock'n'Roll" model

As observed on Figure 3, lift and drag forces create moments on the roughness of the floor, inducing the particle movement according to the moment size.

This competition between adhesion and airflow forces can be expressed by equation (8).

$$
\frac{\mathrm{a}}{2} \mathrm{~F}_{\mathrm{L}}+\mathrm{r}_{\mathrm{p}} \mathrm{F}_{\mathrm{D}}>\mathrm{af} \mathrm{a}_{\mathrm{a}}
$$

Where, $a$ is the distance between the two irregularities on which the particle rests and $r_{p}$ the particle radius. Dividing the inequality (8) by $a$, leads to:

$$
\frac{1}{2} \mathrm{~F}_{\mathrm{L}}+\frac{\mathrm{r}_{\mathrm{p}}}{a} \mathrm{~F}_{\mathrm{D}}>\mathrm{f}_{\mathrm{a}}
$$

According to the experimental data analysed by Reeks \& Hall [14], for micron-size particles deposited on a polished stainless-steel surface, the ratio between the particle radius and the distance between two irregularities is approximately equal to 100 , thus:

$$
\frac{\mathrm{r}_{\mathrm{p}}}{\mathrm{a}} \approx 100
$$

This observation implies that the term including the lift force can, in most cases, be overlooked, even if it is maintained in this study.

Thus, with equations (5), (7), (9) and (10), the balance of airflow forces required to mobilize a spherical particle deposited on a flat surface gives:

$$
\mathrm{F}=\frac{1}{2} \mathrm{~F}_{\mathrm{L}}+\frac{\mathrm{r}_{\mathrm{p}}}{a} \mathrm{~F}_{\mathrm{D}}=2.1 \rho v^{2}\left(\mathrm{~d}_{\mathrm{p}}^{+}\right)^{2.31}+801 \rho v^{2}\left(\mathrm{~d}_{\mathrm{p}}^{+}\right)^{2}
$$

Adhesive forces cannot be simply expressed due to the floor roughness, but Reeks \& Hall [14] supposed that they are distributed according to a log-normal distribution given by:

$$
\varphi\left(\mathrm{f}_{\mathrm{a}}^{\prime}\right)=\frac{1}{\sqrt{2 \pi}} \frac{1}{\mathrm{f}_{\mathrm{a}}^{\prime} \ln \left(\sigma_{\mathrm{a}}^{\prime}\right)} \mathrm{e}^{\left(-\frac{1}{2}\left(\frac{\ln \left(\mathrm{f}_{\mathrm{a}}^{\prime} / \mathrm{f}_{\mathrm{a}}^{\prime}\right)}{\ln \left(\sigma_{\mathrm{a}}^{\prime}\right)}\right)^{2}\right)}
$$

where $\mathrm{f}_{\mathrm{a}}^{\prime}=\mathrm{f}_{\mathrm{a}} / \mathrm{F}_{\text {JKR }}$ represents the local adhesion force $\mathrm{f}_{\mathrm{a}}(\mathrm{N})$ normalised according to the JKR theoretical model, $\overline{\mathrm{f}_{\mathrm{a}}^{\prime}}$ represents the geometric mean of normalised adhesion forces and $\sigma_{\mathrm{a}}^{\prime}$ the geometric standard deviation of the distribution of normalised adhesive forces. 
The JKR model [19] provides a theoretical adhesion force that takes into account particle deformation due to drag on contact edges. It is presented in equation (3).

For the determination of $\overline{\mathrm{f}_{\mathrm{a}}^{\prime}}$ and $\overline{\sigma_{\mathrm{a}}^{\prime}}$, the correlations of Biasi et al. [15] were finally retained and are presented in equations (13) and (14)

With these correlations, the effects of irregularities on the deposit surface and on the particle can be taken into account in the distribution of adhesive forces.

$$
\begin{aligned}
& \overline{\mathrm{f}_{\mathrm{a}}^{\prime}}=0.016-0.0023\left(\frac{\mathrm{d}_{\mathrm{p}}}{2}\right)^{0.545} \\
& \overline{\sigma_{\mathrm{a}}^{\prime}}=1.8-0.136\left(\frac{\mathrm{d}_{\mathrm{p}}}{2}\right)^{1.4}
\end{aligned}
$$

In equations (13) and (14), the particle diameter $d_{p}$ is expressed in micrometer and the data used to establish them corresponds to particle-surface pairs with a surface energy of adhesion close to $0.56 \mathrm{~J} . \mathrm{m}^{-2}$ and particle diameters between $0.1 \mu \mathrm{m}$ and $30 \mu \mathrm{m}$.

As a synthesis of the model, it can be noticed that it is mainly depending on three variables: the friction velocity, the particle diameter and the surface energy of adhesion and most of existing models [20] agree with this observation.

However, knowing the presence of the fluid density in the model and given the specific nature of the LOVA in terms of pressure change, it is necessary to take into account the variation of the fluid density when calculating airflow forces affecting particle resuspension and dispersion models.

The "Rock'n'Roll" model calculates the resuspension rate knowing the constant resuspension specific rate $p$ for an adhesive force value $f_{a}$.

$$
\left\{\begin{array}{c}
p\left(f_{a}\right)=n_{\theta}\left(e^{\left(\frac{-\left(f_{a}-\overline{\mathrm{F}}\right)^{2}}{2 \overline{\mathrm{f}}^{2}}\right)} / \frac{1}{2}\left[1+\operatorname{erf}\left(\frac{\left(\mathrm{f}_{\mathrm{a}}-\overline{\mathrm{F}}\right)}{\sqrt{2 \overline{\mathrm{f}}^{2}}}\right)\right]\right), \text { if } \mathrm{f}_{\mathrm{a}}-\overline{\mathrm{F}} \geq 0.75 \sqrt{\overline{\mathrm{f}^{2}}} \\
\mathrm{p}\left(\mathrm{f}_{\mathrm{a}}\right)=\mathrm{n}_{\theta}, \quad \text { if } \mathrm{f}_{\mathrm{a}}-\overline{\mathrm{F}}<0.75 \sqrt{\overline{\mathrm{f}^{2}}}
\end{array}\right.
$$

Where $n_{\theta}$ is the surface-particle resonance frequency $\left(\mathrm{s}^{-1}\right), \bar{F}$ and $f$ are respectively the mean and fluctuating component of the instantaneous airflow force $\mathrm{F}(t)(\mathrm{N})$; the expression of $\bar{F}$ is given at equation (11), whereas the fluctuation $f$ and the frequency $n_{\theta}$ can be calculated by the following equations, using the experimental measurements carried out by Hall [21]: 


$$
\begin{aligned}
& f=0.2 \bar{F} \\
& n_{\theta}=0.00658\left(\frac{u^{*^{2}}}{v}\right)
\end{aligned}
$$

From the constant resuspension specific rate $p\left(f_{a}\right)$, the resuspension rate $\lambda(t)$, depending essentially on the particle diameter and the shear velocity, can be calculated according to airflow conditions: constant or variable.

\subsection{Model for a constant shear velocity}

Knowing the expressions of the constant resuspension specific rate $p$ and the adhesive force distribution $\varphi$, the resuspension rate $\lambda(t)$ for constant airflow conditions, involving a constant shear velocity, can be calculated from the following expression proposed by Reeks \& Hall [14] in the "Rock'n'Roll" model:

$$
\lambda(t)=\int_{0}^{\infty} \varphi\left(f_{a}^{\prime}\right) p\left(f_{a}^{\prime}\right) e^{-p\left(f_{a}^{\prime}\right) t} d f_{a}^{\prime}
$$

In order to obtain $p\left(f_{a}^{\prime}\right), f_{a}$ must be replaced by $\left(f_{a}^{\prime} \cdot F_{J K R}\right)$ in equation (15).

Figure 4 shows the time evolution of $\lambda(t)$ for a particle diameter of $10 \mu \mathrm{m}$ and a shear velocity of $0.5 \mathrm{~m} \cdot \mathrm{s}^{-1}$.

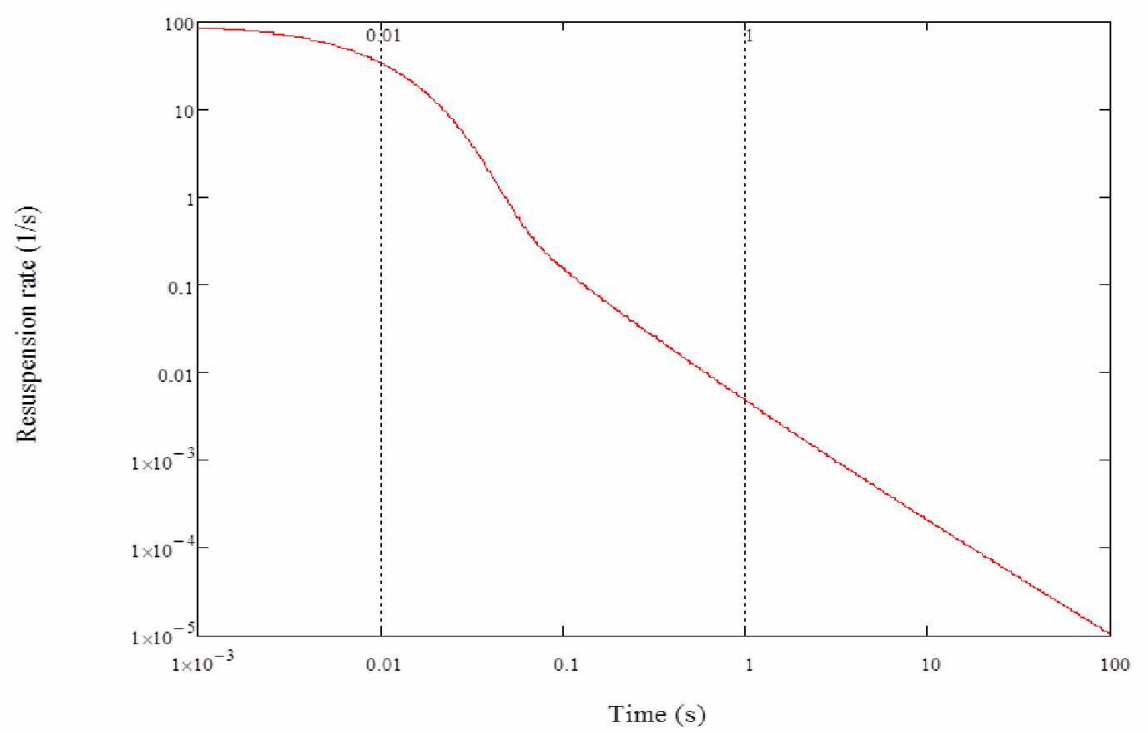

Figure 4 - time evolution of the resuspension rate for a constant shear velocity

$$
\left(d_{p}=10 \mu \mathrm{m}, \mathrm{u}^{*}=0.5 \mathrm{~m} \cdot \mathrm{s}^{-1}\right)
$$


Figure 4 shows that, at the beginning, the resuspension rate decreases quickly: this phase corresponds to the short term resuspension. After $0.1 \mathrm{~s}$, the resuspension rate is three decades lower than at the initial time. Then, this rate decreases more slowly: this phase is the long term resuspension.

Equation (18) is relevant for constant airflow conditions, but many applications exist with variable airflow conditions. Hence, another expression is necessary to take into account the transient phenomenon.

\subsection{Adaptation of the model for a variable shear velocity}

To take into account the transient configuration, it is necessary to discretize the timescale with a timestep $\Delta t$ small enough for considering a constant shear velocity on each time step. This adaptation was proposed by Choi et al. [22] in a study on the particle resuspension during a footstep. The mean value of the resuspension rate $\bar{\lambda}(\mathrm{t})$ is thus calculated for the timestep $\Delta t$ considered by the following expression:

$$
\bar{\lambda}(\mathrm{t})=\frac{1}{\Delta t} \int_{0}^{\Delta t} \lambda(\mathrm{t}) d t=\frac{1}{\Delta t} \int_{0}^{\infty} \varphi\left(\mathrm{f}_{\mathrm{a}}^{\prime}\right)\left(1-\mathrm{e}^{-\mathrm{p}\left(\mathrm{f}_{\mathrm{a}}^{\prime}\right) \Delta \mathrm{t}}\right) \mathrm{df}_{\mathrm{a}}^{\prime}
$$

As an example, Figure 5 shows the evolution of the mean value of the resuspension rate according to the timestep for 1 micron alumina particles and a friction velocity of $2 \mathrm{~m} \cdot \mathrm{s}^{-1}$. It highlights the influence of the timestep on this value. Indeed, if the timestep is too large, the mean value of the resuspension rate can be widely underestimated.

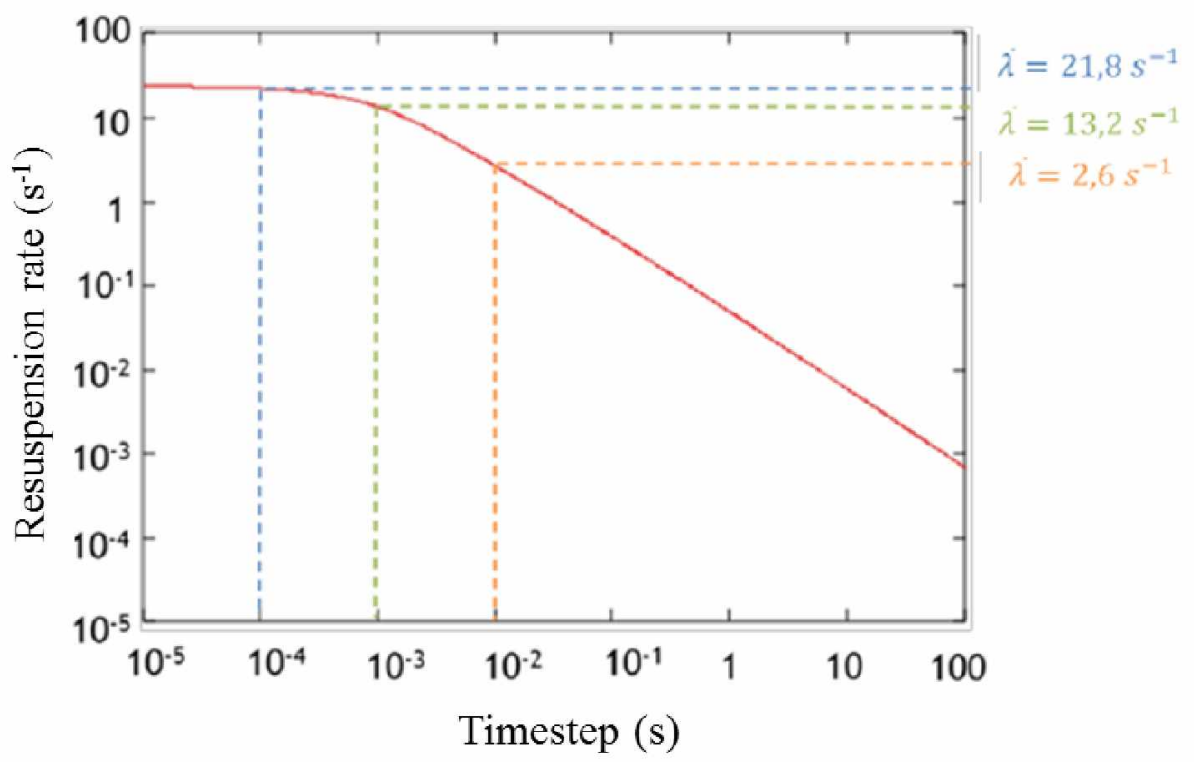

Figure 5 - resuspension rate evolution according to the timestep $\left(d_{p}=1 \mu \mathrm{m}, \mathrm{u}^{*}=2 \mathrm{~m} \cdot \mathrm{s}^{-1}\right)$ 
From Figure 5, it can be deduced that below a timestep of $10^{-3} \mathrm{~s}$, the evolution of the resuspension rate is less than a factor 2 , which is reasonable, but above $10^{-3} \mathrm{~s}$, the factor can reach many decades. Thus, the choice of the timestep is crucial.

\section{IMPLEMENTATION OF THE ROCK'N ROLL MODEL IN ANSYS CFX}

The "Rock'n'Roll" model was implemented in ANSYS CFX in order to take into account the resuspension phenomenon together with aerosol transport and deposition calculations allowing to assess the airborne particle amount for industrial issues of dust explosion or of dust inhalation by operators.

For this implementation, the addition of a source term to the particle transport equation was necessary:

$$
\frac{\partial \rho \mathrm{Y}_{\mathrm{p}}}{\partial \mathrm{t}}+\nabla \cdot\left(\rho \mathbf{U} \mathrm{Y}_{\mathrm{p}}\right)=\nabla \cdot\left(\left(\rho \mathrm{D}+\frac{\mu_{\mathrm{t}}}{\mathrm{Sc}_{\mathrm{t}}}\right) \nabla \mathrm{Y}_{\mathrm{p}}\right)+\mathrm{S}_{\mathrm{p}}
$$

Where $Y_{p}$ is the particle mass fraction, $\rho$ is the carrier fluid density $\left(\mathrm{kg} \cdot \mathrm{m}^{-3}\right), \mathbf{U}$ is the mean fluid velocity vector $\left(\mathrm{m} . \mathrm{s}^{-1}\right), \mathrm{D}$ is the particle diffusion coefficient $\left(\mathrm{m}^{2} \cdot \mathrm{s}^{-1}\right), \mu_{\mathrm{t}}$ is the eddy viscosity $($ Pa.s), $\mathrm{Sc}_{\mathrm{t}}$ is the turbulent $\mathrm{Schmidt}$ number and $\mathrm{S}_{\mathrm{p}}$ is the global particle source term $\left(\mathrm{kg} \cdot \mathrm{m}^{-3} \cdot \mathrm{s}^{-1}\right)$.

The resuspension rate $\lambda(\mathrm{t})$ including Biasi's model for the adhesive forces distribution is thus implemented in the global source term $S_{p}$, associated to the local particle surface concentration $\mathrm{C}_{\mathrm{s}}(\mathrm{t})\left(\mathrm{kg} \cdot \mathrm{m}^{-3}\right)$ :

$$
\mathrm{S}_{\mathrm{p}, \mathrm{res}}=\lambda(\mathrm{t}) \mathrm{C}_{\mathrm{s}}(\mathrm{t})
$$

As the particles are resuspended, the local particle surface concentration has to decrease over time by this way. Hence, a variable associated to the surface concentration has also been implemented in order to take into account this evolution.

$$
\left\{\begin{array}{c}
\mathrm{C}_{\mathrm{s}}(\mathrm{t}+\Delta \mathrm{t})=\mathrm{C}_{\mathrm{s}}(\mathrm{t})(1-\lambda(\mathrm{t}) \Delta t), \text { if } u^{*} \text { is constant } \\
\mathrm{C}_{\mathrm{s}}(\mathrm{t}+\Delta \mathrm{t})=\mathrm{C}_{\mathrm{s}}(\mathrm{t})(1-\bar{\lambda}(\Delta \mathrm{t}) \Delta t), \text { if } u^{*} \text { is variable }
\end{array}\right.
$$

Both types of model, either for a constant or variable shear velocity, have thus been implemented in ANSYS CFX and their validation has been conducted using the experimental data. Furthermore, it can be noticed that we included in ANSYS CFX a specific model for the particle transport and deposition, developed and validated by Nerisson et al. [23]. The transport model is integrated to the transport equation (20), whereas the deposition model is implemented by the mean of a sink term 
$S_{p \text {,dep }}$ removed from the resuspension $S_{p, r e s}$ in the global source term $S_{p}$. Furthermore, this deposition flux $S_{p, d e p}$ is added to the surface concentration in order to take into account an enrichment by the deposition.

\section{EVALUATION OF THE ROCK'N ROLL MODEL IMPLEMENTATION IN ANSYS CFX}

\subsection{Constant shear velocity case}

\section{- Experimental study description}

The evaluation of the model for a constant shear velocity has been performed with experimental data acquired on an analytical bench called "BISE" dedicated to the study of resuspension. The work is related to the resuspension of Alumina (SPM95) particle and Carbon particle collected in the Tore Supra Tokamak [24] [25]. Figure 6 shows the "BISE" device.

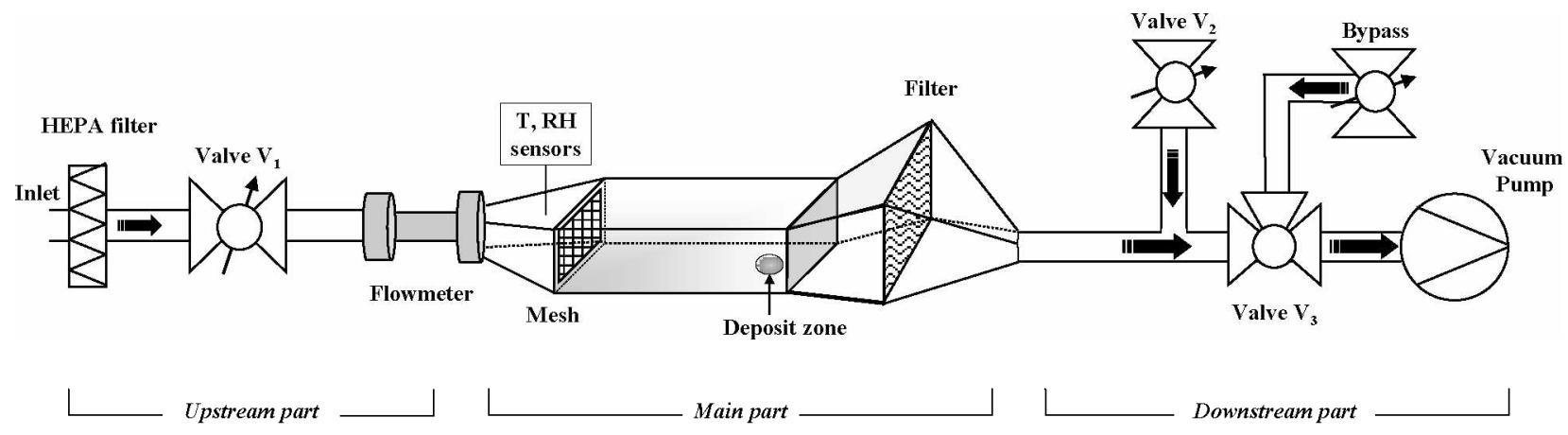


Figure 6 - analytical bench for resuspension

The bench is composed of a rectangular channel with a length, a width and a height of $40 \mathrm{~cm}, 12 \mathrm{~cm}$ and $7 \mathrm{~cm}$ respectively; this bench is divided into three parts: the upstream zone, the main zone and the downstream zone. The main zone is the test section where a one-layer deposit of powder is dropped off. This zone is swept by a controlled constant airflow ensuring a constant shear velocity. The other zones contain all the instrumentation allowing to control the flow and to measure the resuspended particle parameters (diameter, concentration...).

The experimental and numerical resuspension coefficient $K_{r}$ can be now compared and, for that, the experimental resuspension coefficient is calculated by the following expression:

$$
\mathrm{K}_{\mathrm{r}}=\frac{\Delta m}{m_{i}}
$$

where $m_{i}$ is the particle deposit mass at the initial time $(\mathrm{g})$ and $\Delta m$ is the particle deposit mass lost (thus resuspended) during the test (g).

Many experiments have been performed for different types of powder with different particle sizes and for different airflow velocities [25].

Two experimental configurations are retained and are detailed in Table 1.

Table 1 - particle characteristics for the resuspension test in BISE

\begin{tabular}{|c|c|c|c|c|c|}
\hline Configuration & Powder & $\begin{array}{c}\text { Geometric mean } \\
\text { diameter }(\mu \mathrm{m})\end{array}$ & $\begin{array}{c}\text { Geometric } \\
\text { standard } \\
\text { deviation }\end{array}$ & $\begin{array}{c}\text { Free-stream } \\
\text { Velocity }\left(\mathrm{m} . \mathrm{s}^{-1}\right)\end{array}$ & $\begin{array}{c}\text { Adhesion } \\
\text { surface energy } \\
\left(\mathrm{J} . \mathrm{m}^{-2}\right)\end{array}$ \\
\hline 1 & $\begin{array}{c}\text { Alumina } \\
\text { SPM95 }\end{array}$ & 6.4 & 1.48 & 6.0 to 10.0 & $0.56[26]$ \\
\hline 2 & $\begin{array}{c}\text { Carbon Tore } \\
\text { Supra }\end{array}$ & Mode $1: 1.4$ & 1.7 & 4.0 to 10.0 & $4.4[27]$ \\
\hline
\end{tabular}

- CFD study description

For the CFD study, the geometry of the "BISE" facility is built with ANSYS Design Modeler and is presented in Figure 7. 


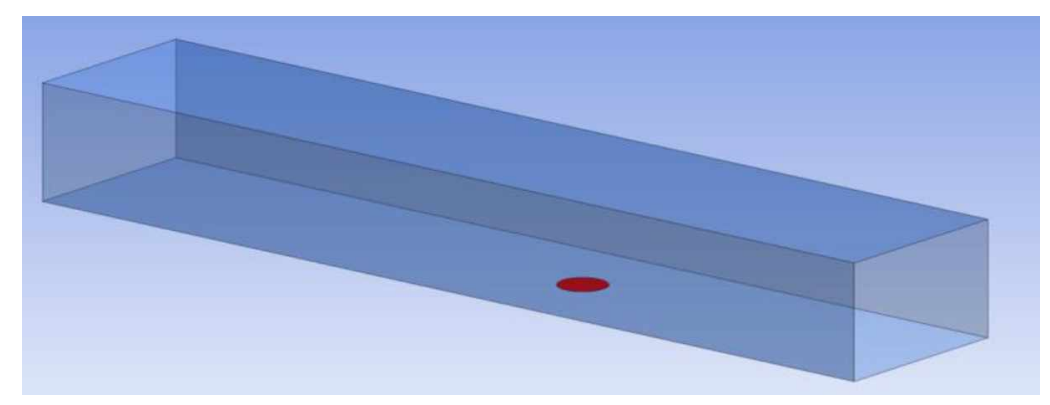

Figure 7 - geometry of the "BISE" device

The simulations are launched with this geometry by using the k- $\omega$ SST (Shear Stress Transport) turbulence model, which was chosen after a sensitivity study described by Gelain et al. [12]. This model remains a good compromise between accuracy and calculation time. The time duration is $15 \mathrm{~min}$ with a time step of $10^{-3} \mathrm{~s}$ during $0.1 \mathrm{~s}$, then $0.1 \mathrm{~s}$ up to the end of the calculation. As shown by a red surface in Figure 7 , the floor surface concentration is initialized in order to allow the particle resuspension. For that, a concentration source term is applied in the first cell of the mesh close to the floor. The numerical resuspension coefficient is exactly calculated with the same method as the experimental one, by monitoring the mass loss during the test. The numerical results are then compared to those of the analytical form of the Rock ' $n$ Roll model, in order to validate the implementation of the model in the ANSYS CFX code, and especially the use of the resuspension rate in its discretized form presented in figure 5, according to the calculation timestep used in ANSYS CFX.

\section{- Model evaluation}

The comparison between analytical and CFD results of resuspension coefficients is presented in Figure 8 which represents the resuspension coefficient values according to the studied velocities. In this figure are also presented the experimental results for both tested powders (SPM95 and Tore Supra). 


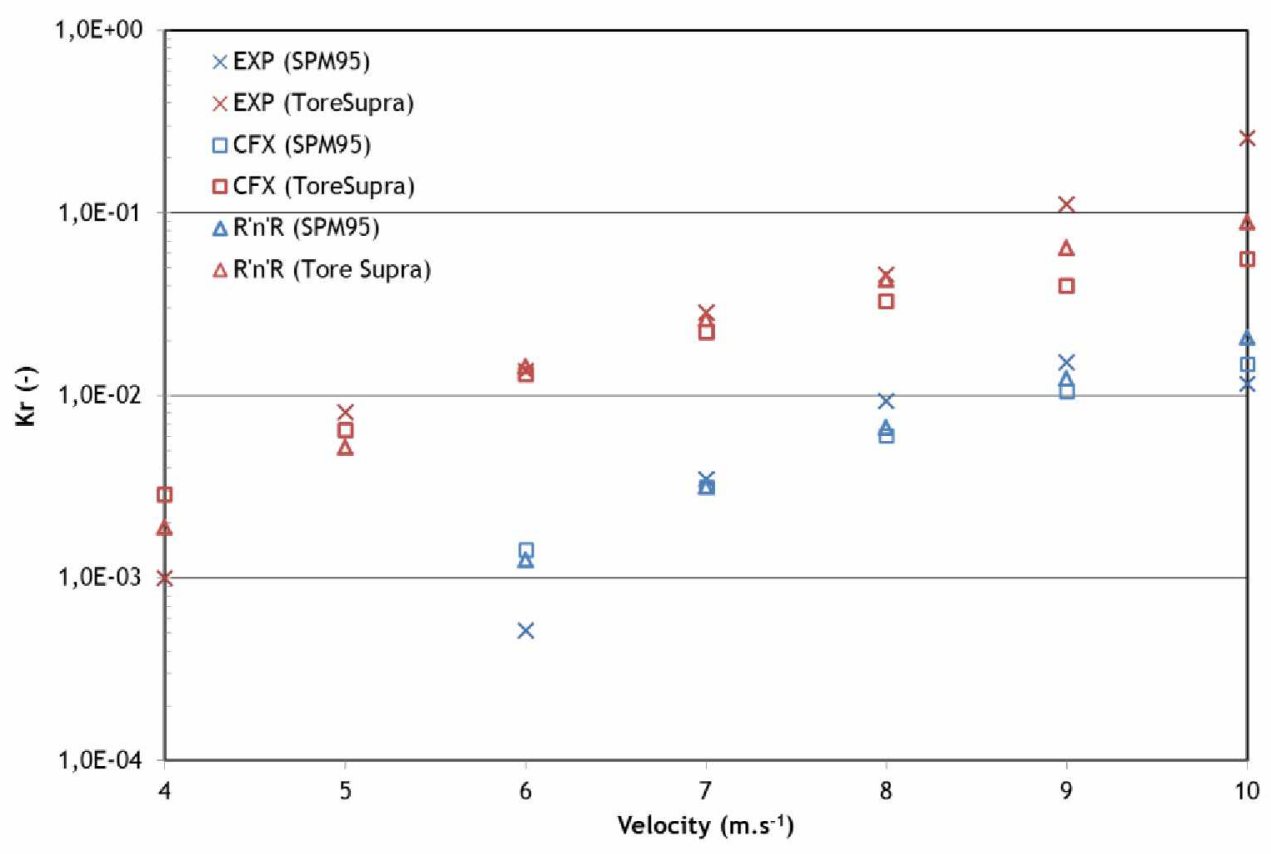

Figure 8 - comparison between experimental, analytical and ANSYS CFX results of resuspension coefficients

As expected, the resuspension coefficients increase with the velocity (hence with the shear velocity). It can be noticed that the resuspension coefficients of the Tore Supra particles are greater than those of the SMP95 particles due to larger particle diameters, knowing that for a constant velocity, the larger the particles, the greater the resuspension.

Furthermore, Figure 8 shows a satisfactory agreement between analytical solution of the Rock'n'Roll model giving the resuspended fraction and numerical results obtained by ANSYS CFX using the Rock'n'Roll resuspension rate with the discretization presented in Figure 5. These results strengthen the implementation in ANSYS CFX of the Rock'n'Roll model for a constant shear velocity.

It can be also precise that $2 / 3$ of the results obtained with CFD calculations have a deviation with the experimental ones inferior to $35 \%$. The minimal deviation is $4.5 \%$ and the maximal deviation is $187 \%$.

\subsection{Variable shear velocity case}

\section{- Experimental study description}

The evaluation of the model for a variable shear velocity requires to carry out experiments with a controlled variable airflow that can be reproduced. For that, an experiment has been designed [28], consisting in the fall of a plate, with its own weight, on a surface covered with alumina particles 
deposit in a ventilated enclosure. In this latter, particles are dropped off on a defined surface of the floor under the plate, the plate is falling and the resuspended particles are entrained by the airflow and measured at the enclosure outlet. The experiment device is presented in Figure 9.

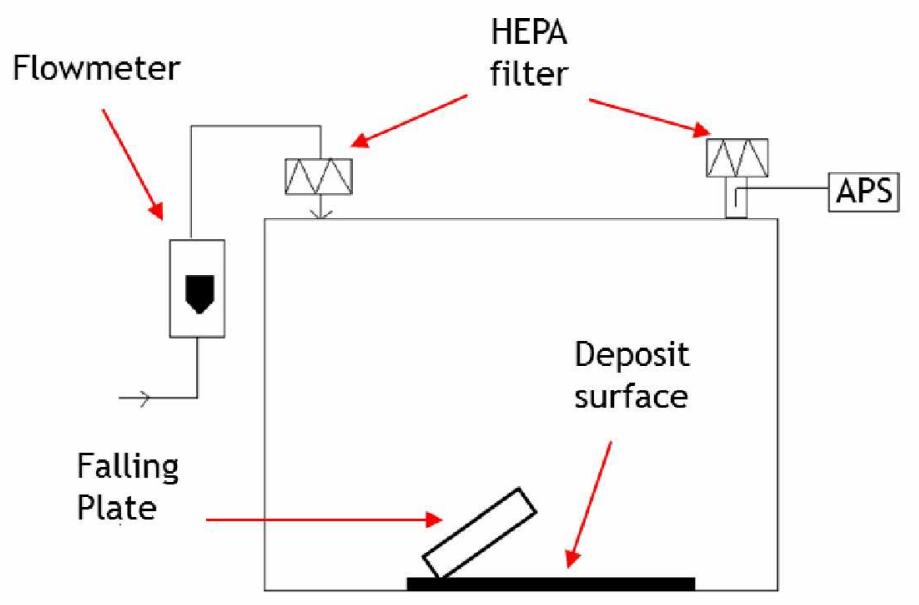

Figure 9 - scheme of the experimental setup for the resuspension with a variable shear velocity Several resuspension experiments were performed and the concentration time evolutions for some of them are presented in Figure 10 for 1 micron particles.

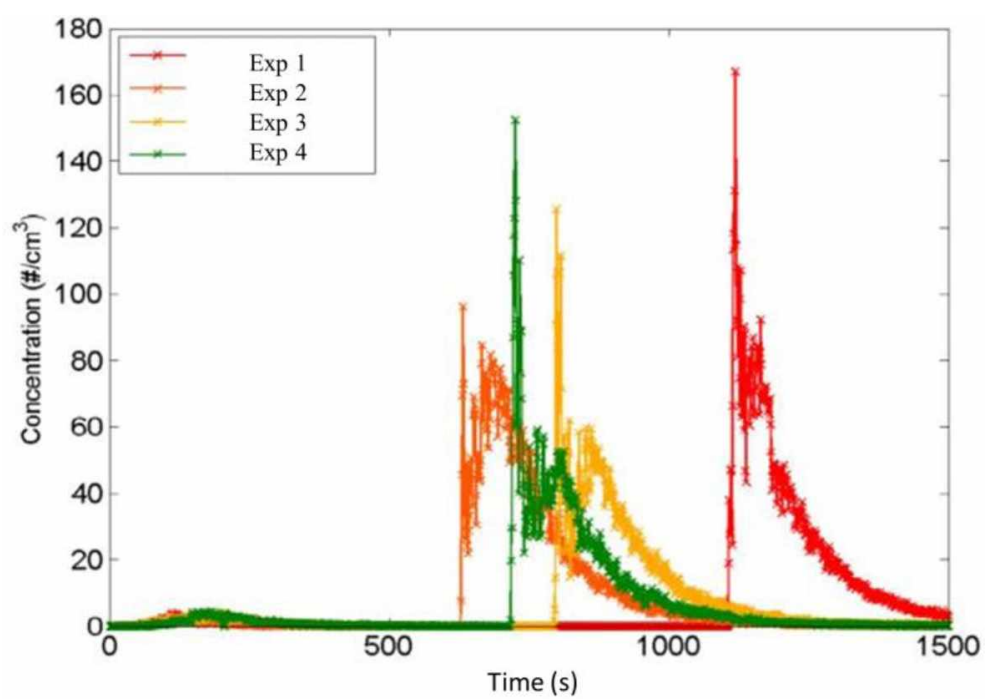

Figure 10 - concentration time evolution at the enclosure outlet $\left(\mathrm{d}_{\mathrm{amm}}=1 \mu \mathrm{m}\right)$

All the concentration curves show a peak, probably at the maximum of the shear velocity, when the plate is close to the floor, then the concentration decreases slowly until the enclosure is empty. To calculate the resuspension coefficient, the following expression is used:

$$
\mathrm{K}_{\mathrm{r}, \exp }=\frac{\mathrm{R}_{\mathrm{t}} \mathrm{V} \int_{0}^{\infty} \mathrm{C}_{\mathrm{vol}} \mathrm{dt}}{\mathrm{C}_{\text {surf }} S_{\text {plate }}}
$$


Where $C_{v o l}$ is the particle volume concentration $\left(\mathrm{kg} \cdot \mathrm{m}^{-3}\right), t$ is the time $(\mathrm{s}), \mathrm{R}_{\mathrm{t}}$ is the sum of the enclosure air exchange rate and the particle deposit rate $\left(\mathrm{s}^{-1}\right), \mathrm{V}$ is the enclosure volume $\left(\mathrm{m}^{3}\right), \mathrm{C}_{\text {surf }}$ is the surface concentration $\left(\mathrm{kg} \cdot \mathrm{m}^{-2}\right)$ and $S_{\text {plate }}$ the plate surface $\left(\mathrm{m}^{-2}\right)$.

\section{- CFD study description}

The experiment described above has been reproduced by CFD calculations. The geometries of the enclosure and the plate were designed with Ansys Design Modeler (Figure 11).
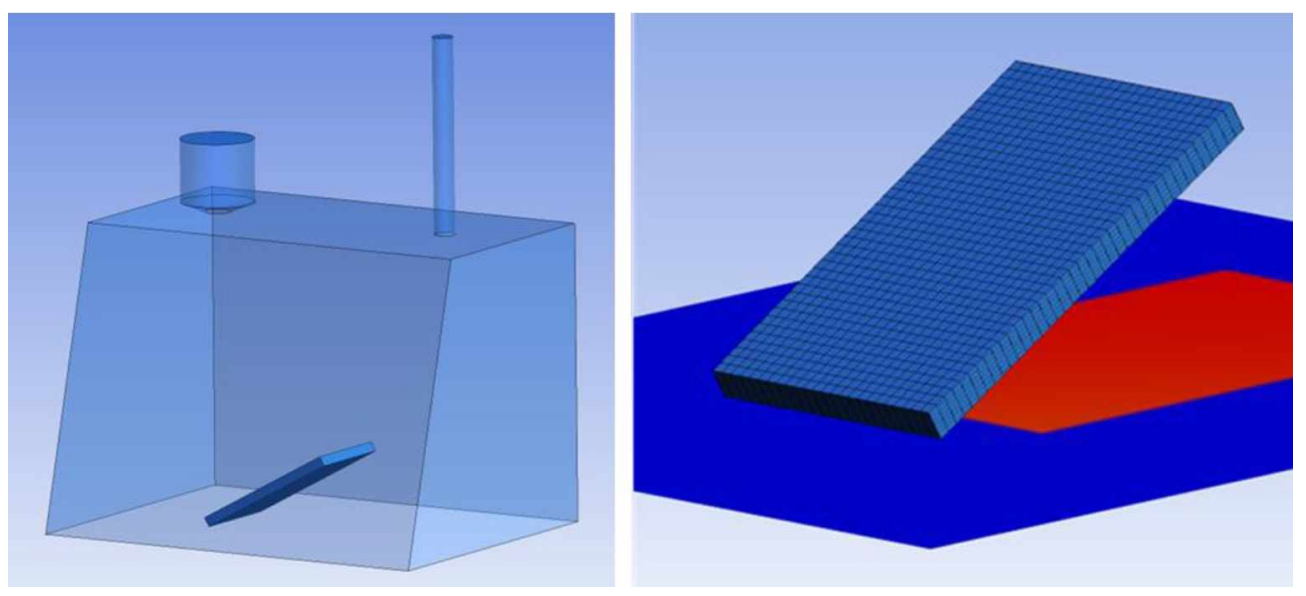

Figure 11 - geometries of the enclosure with the plate

The geometry is meshed with a hybrid mesh (tetrahedral-hexahedral) including 800000 elements and the simulations are launched by using the k- $\omega$ SST (Shear Stress Transport) turbulence model. The fall of the plate is performed by using an immersed solid method and by considering the following fall angular velocity $U_{\theta}$ equation depending on the angular acceleration $A_{\theta}$ defined from the plate dimensions and the gravity:

$$
\begin{aligned}
& \mathrm{U}_{\theta}=A_{\theta} t \\
& A_{\theta}=\frac{3}{2} g \frac{L_{p}}{\frac{l_{p}^{2}}{4}+L_{p}^{2}}
\end{aligned}
$$

where $L_{p}$ is the plate length $(\mathrm{m}), l_{p}$ is the plate width $(\mathrm{m}), \mathrm{g}$ is the gravity $\left(\mathrm{m} . \mathrm{s}^{-2}\right)$ and $\mathrm{t}$ is the time (s).

The time duration is defined by the fall time depending on the angular acceleration and the initial angle between the plate and the floor. When the plate altitude is at $0 \mathrm{~m}$, the plate stops to fall and the calculation of the airflows goes on until the enclosure is emptied. The timestep is $10^{-2} \mathrm{~s}$ during 
$0.14 \mathrm{~s}$, then $10^{-4} \mathrm{~s}$ when the plate approaches the floor and moves away up to $0.16 \mathrm{~s}$, then the timestep increases progressively up to $2 \mathrm{~s}$.

The resuspension coefficient determination can be made by monitoring the resuspended particle mass flowrate during the fall of the plate and by calculating the expression:

$$
\mathrm{K}_{\mathrm{r}, \mathrm{cfx}}=\frac{\int_{0}^{t_{t}} \mathrm{q}_{\mathrm{m}} \mathrm{dt}}{\mathrm{m}_{\mathrm{s}}}
$$

where $\mathrm{q}_{\mathrm{m}}$ is the resuspended particle mass flowrate $\left(\mathrm{kg} \cdot \mathrm{s}^{-1}\right), \mathrm{m}_{\mathrm{s}}$ is the total mass of particle deposited on the floor $(\mathrm{kg})$ and $t_{t}$ is the total simulated time (s).

For these calculations, an adhesion surface energy of $0.56 \mathrm{~J} . \mathrm{m}^{-2}$ has been considered, according to the preconisation of Biasi [26].

\section{- Model validation}

The numerical results were then compared to experimental ones in order to evaluate the implementation of the model in the ANSYS CFX code. All experimental measurements of the concentration evolutions were averaged in order to obtain only one experimental average evolution and one mean resuspended fraction.

First, the time evolutions of the resuspended particle mass concentration are represented in Figure 12. Despite the experimental oscillations, the agreement with the CFD results is reasonable, with a large peak at the beginning of the resuspension, followed by a quick decrease during $30 \mathrm{~s}$ and finally a slow decrease up to the end of the test. The concentration is normalized because the amount of particle deposited on the floor is not the same between the experiments and the numerical calculations. 


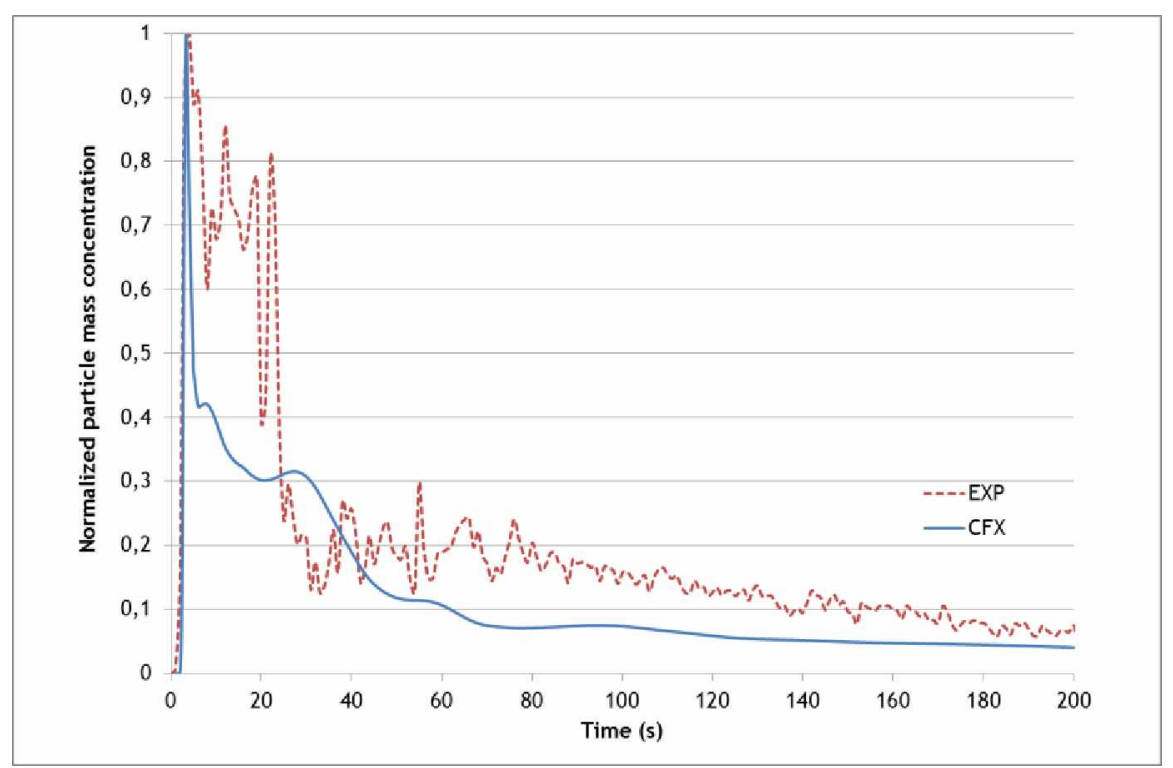

Figure 12 - normalized particle mass concentration evolution during the test $\left(\mathrm{d}_{\mathrm{amm}}=1 \mu \mathrm{m}\right)$

The average resuspension coefficient is then calculated for all experimental cases. For particles with an aerodynamic median diameter (AMMD) of $1 \mu \mathrm{m}$, average resuspension coefficients of $6.10^{-3} \pm 2.10^{-3}$ and $4.10^{-3}$ are found for the experimental case and the CFX case respectively.

This good agreement between the experimental and numerical results allow to ensure that the implementation of the Rock'n'Roll model for a variable shear velocity in ANSYS CFX is correct and that its use is relevant for an application to the case of a LOVA in ITER, in the continuity of the paper of Gelain et al. [12] concerning the airflows in ITER in the event of a LOVA.

This comparison allows to ensure that the implementation of the Rock'n'Roll model for a variable shear velocity in ANSYS CFX is correct and that its use is relevant for an application to transient airflow such the case of a LOVA in a vaccum vessel, in the continuity of the paper by Gelain et al. [12] concerning the airflows and shear velocities mapping in a toroidal vessel in the event of an air ingress. 


\section{APPLICATION TO THE CASE OF A LOVA IN A TOROIDAL GEOMETRY}

Before presenting this application, it has to be reminded that the Rock'n'Roll resuspension model was developed with certain limitations: deposited particles are assumed to be spherical, uniformly distributed on the floor, with a monolayer configuration and no interaction between particles. Moreover, this resuspension model does not take into account the reduction of drag force on particles due to molecules "slip" at high Knudsen number.

In a real ITER application, all these conditions are obviously not encountered, but in our application thereafter, the goal is not to be representative of ITER conditions but to demonstrate the feasibility of this kind of calculations in LOVA conditions (temperature, low pressure), with the respect of the applicability of the Navier-Stokes equations, and to study and understand the contribution of the different mechanisms leading to the existence of a dust cloud.

\subsection{CFD simulation description}

To evaluate the resuspension of different particle sizes from the airflows generated during a LOVA, numerical simulations were performed using ANSYS CFX software, into which the resuspension model with a variable shear velocity is implemented.

The first step consists in representing the geometry of the torus with the duct that simulates the breach, which is the source of the loss of vacuum.

For the computational domain, a toroidal geometry is retained with a D-shaped section, the dimensions of which are given in Table 2; they were chosen based on those used in a previous study of Gelain et al. [12].

To represent the break, a circular duct with a diameter of $0.16 \mathrm{~m}$ and a length of $2 \mathrm{~m}$ is used. The duct is located halfway up the torus and is initially closed at the torus wall. Erreur : Source du renvoi introuvable. presents a sectional view of the shape of the torus with its dimensions.

Table 2 - overall dimensions of the torus

\begin{tabular}{|l|c|}
\hline Torus volume & $1,300 \mathrm{~m}^{3}$ \\
\hline Inside radius of torus & $3.99 \mathrm{~m}$ \\
\hline Outside radius of torus & $8.53 \mathrm{~m}$ \\
\hline Height of torus & $9.62 \mathrm{~m}$ \\
\hline
\end{tabular}




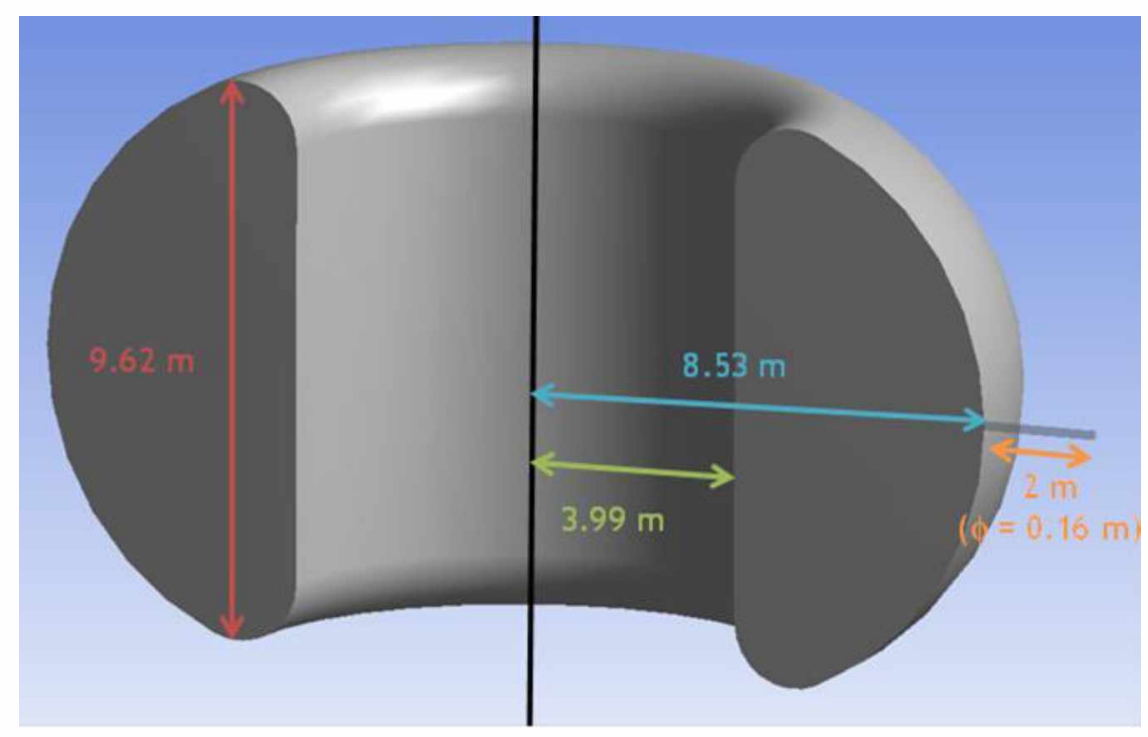

Figure 13 - geometry used for the calculations

The geometry is then discretized in tetrahedral elements as presented on Figure 14, which presents different views of the mesh; its characteristics are summarized in Table 3.

Table 3 - characteristics of the meshing

\begin{tabular}{|l|c|}
\hline Element type & Tetrahedra - Hexahedra \\
\hline Min size & $1.5 \cdot 10^{-3} \mathrm{~m}$ \\
\hline Max size & $0.15 \mathrm{~m}$ \\
\hline Number of elements & $2,557,750$ \\
\hline
\end{tabular}

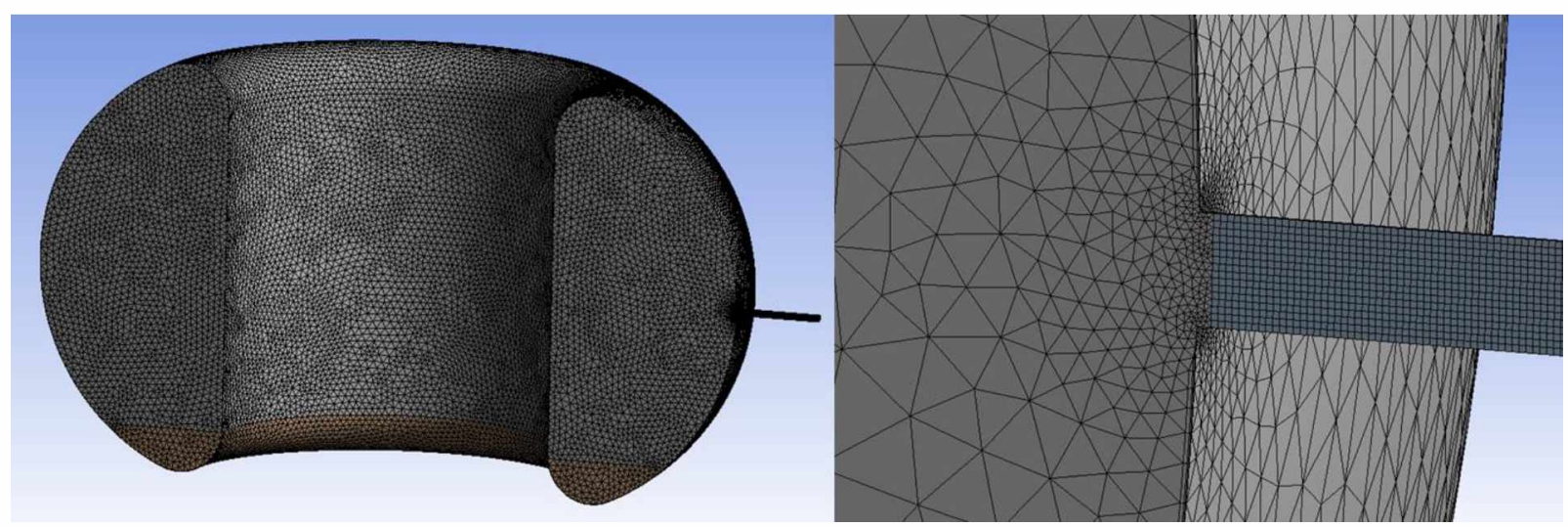

Figure 14 - mesh used for the calculations

The dataset parameters for the calculations are given in Table 4. The k- $\omega$ SST turbulence model was chosen, as for the airflow study presented by Gelain et al. [12].

The airflow parameters used for the calculations were set for a LOVA-type accident scenario as described by Xiao et al. [29] and already used by Gelain et al. [12]. They are given in Table 4 . 
Table 4 - Parameters adopted for modelling with ANSYS CFX software

\begin{tabular}{|c|c|}
\hline Inlet pressure & $10^{5} \mathrm{~Pa}$ \\
\hline Inlet air temperature & $298 \mathrm{~K}$ \\
\hline Initial torus pressure & $800 \mathrm{~Pa}$ \\
\hline Imposed torus wall temperature & $673 \mathrm{~K}$ \\
\hline Initial gas temperature & $493 \mathrm{~K}$ \\
\hline Particle diameters & $\begin{array}{c}2-5-10 \text { microns (corresponding to } 8.8,22 \text { and } 44 \mu \mathrm{m} \\
\text { aerodynamic diameters for tungsten particles) }\end{array}$ \\
\hline Turbulence models & $\mathrm{k}-\omega \mathrm{SST}$ \\
\hline Time step & Adaptive from $10^{-6}$ second to 0.1 second \\
\hline Duration & 20 seconds \\
\hline
\end{tabular}

The surface energy used for these calculations is equal to $4.4 \mathrm{~J} \cdot \mathrm{m}^{-2}$, as preconized by Rondeau [27], from a fit of the Biasi correlation on its experimental data of resuspended fraction acquired in the AUG tokamak. However, Peillon [17] demonstrated from AFM measurements of tungsten spherical particles on a tungsten substrate that the adhesive forces can be very low, leading to surface energy lower than unity.

The initial pressure of $800 \mathrm{~Pa}$ considered for this scenario is far from the real conditions during steady state operation of a tokamak, but as explained before, the goal is not to be representative of ITER operating condition. Furthermore, a LOVA can also occur at any pressure during operations, when a tokamak is set from vacuum to ambient pressure and inversely.

Figure 15 shows the concentration source term applied on the floor, allowing the particle resuspension.

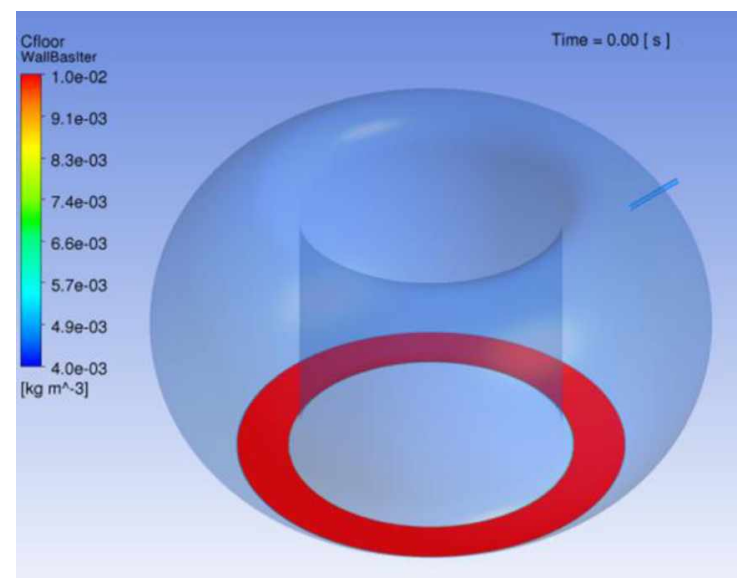

Figure 15 - floor surface initial concentration 
During the calculation, the resuspension coefficient $K_{\text {res }}$ (defined by equation (27)) and the resuspension rate $\lambda_{\text {res }}$ (defined by equation (18)) were monitored as well as the other usual variables such as the friction velocity, the particle mass fraction, the pressure or the floor surface concentration.

It can be noticed that the calculation was stopped at $20 \mathrm{~s}$ corresponding to a vessel pressure around 120 mbar, because the friction velocities on the floor were too low to induce the particle resuspension.

In the next section, the simulation results are presented and discussed in terms of airflows, particle resuspension and dispersion.

\subsection{Simulation results}

\section{- Airflows}

The resuspension is essentially driven by the airflows and more precisely by the friction velocity. Hence, Figure 16 presents the time evolution of the friction velocity on the floor where the particles are dropped off. As seen on this figure, the maximal friction velocity reaches around $8 \mathrm{~m} / \mathrm{s}$ for a mean value of around $4 \mathrm{~m} / \mathrm{s}$.

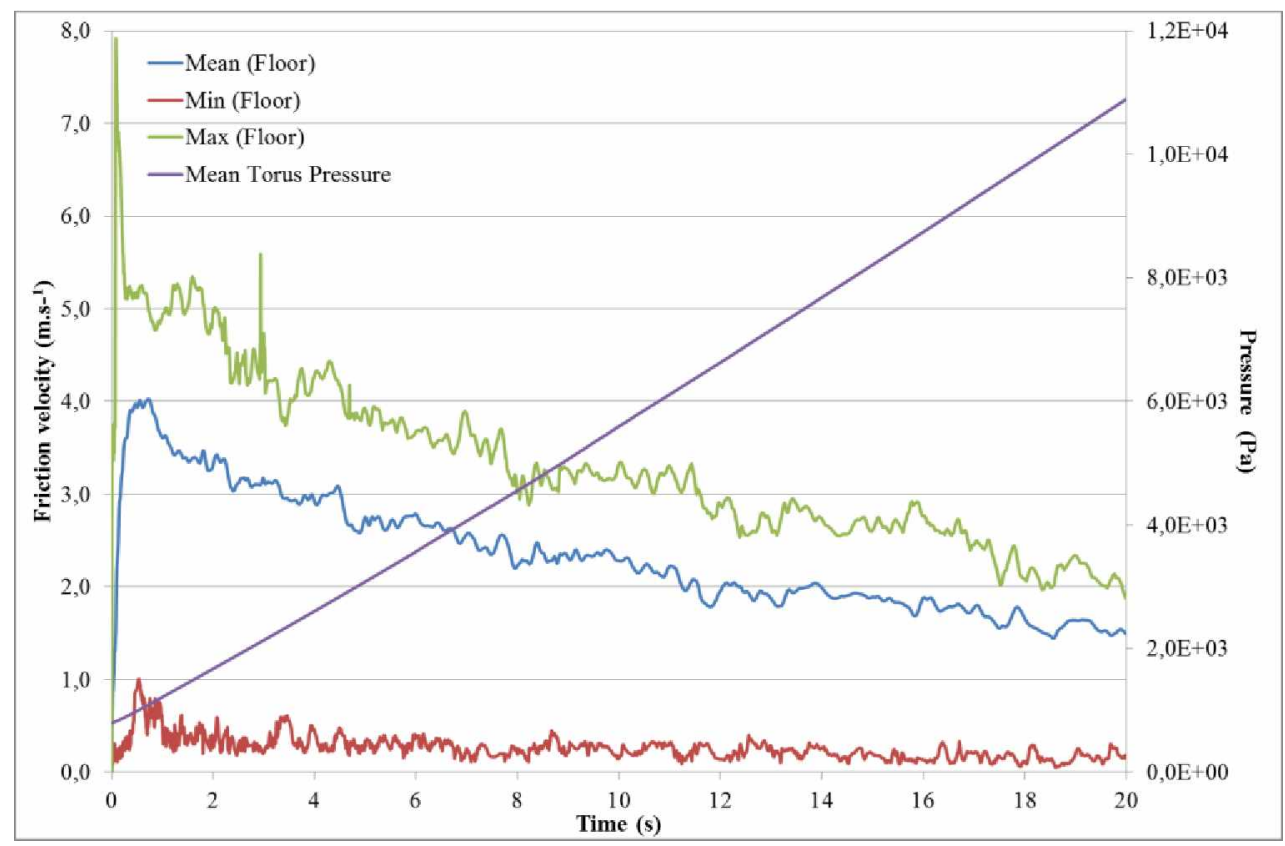

Figure 16 - friction velocity on the floor and pressure inside the torus

Figure 17 shows the friction velocity fields on the floor at different times that allows to visualize its distribution. At $0.1 \mathrm{~s}$, the friction velocity is maximal close to the air entry and below the impaction with the wall. At $1 \mathrm{~s}$, the friction velocities are more distributed around the wall of the torus core and the maximal velocity began to decrease. For the other times, the friction velocities are almost 
totally distributed all around the torus and are quite low compared to the maximal friction velocity at $0.1 \mathrm{~s}$.
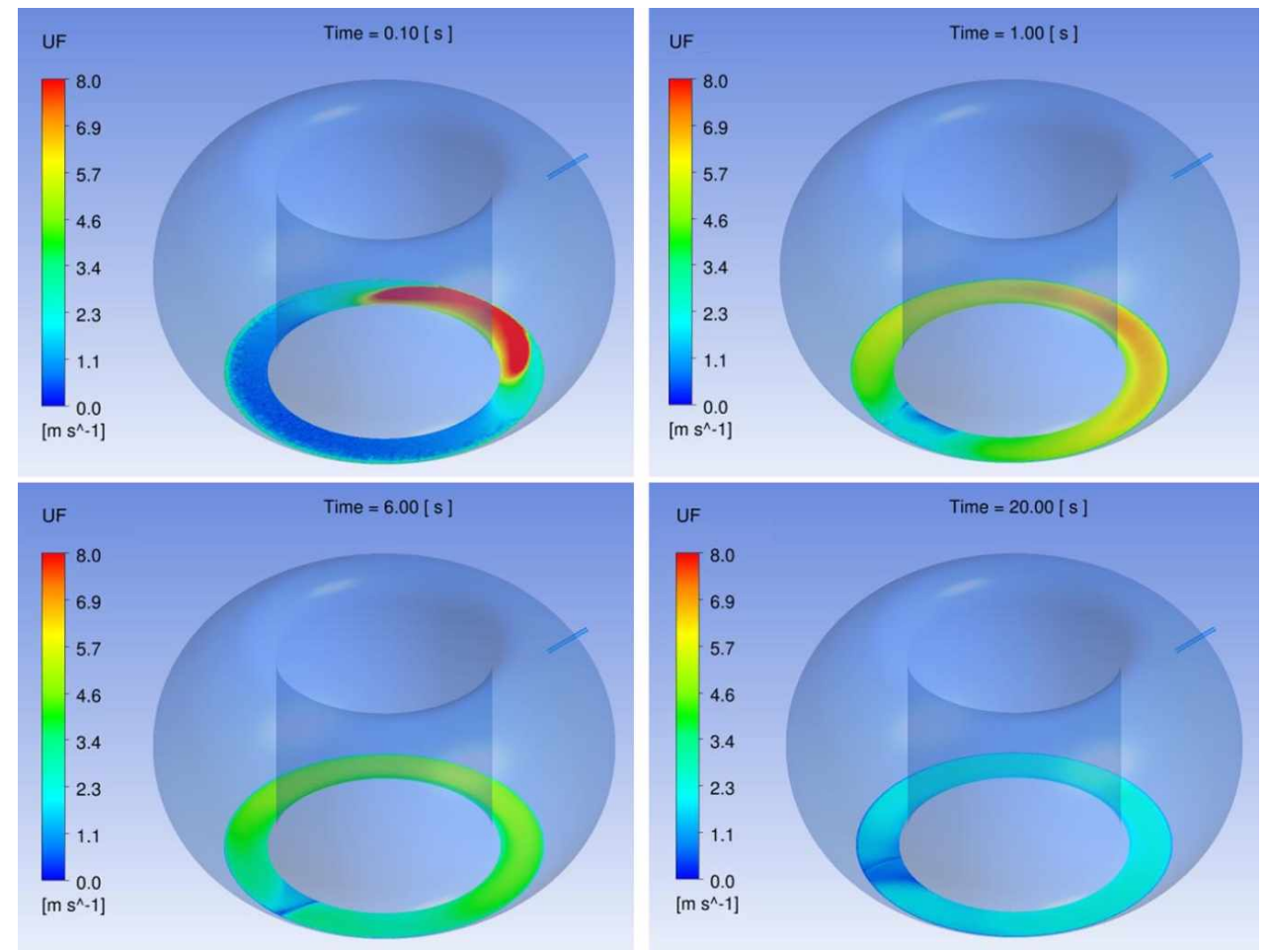

Figure 17 - friction velocity fields on the floor

Taking into account this time evolution of the friction velocity distribution in the torus, a link can be established with the time evolution of the resuspension as described in the next section.

Finally, Figure 18 shows the airflow streamlines inside the torus. The geometry and the strong velocities induce vortices which will entrain the particles along the torus walls and disperse them inside the torus. The observation of these vortices is very important to understand how the particles are dispersed as shown in the next section.

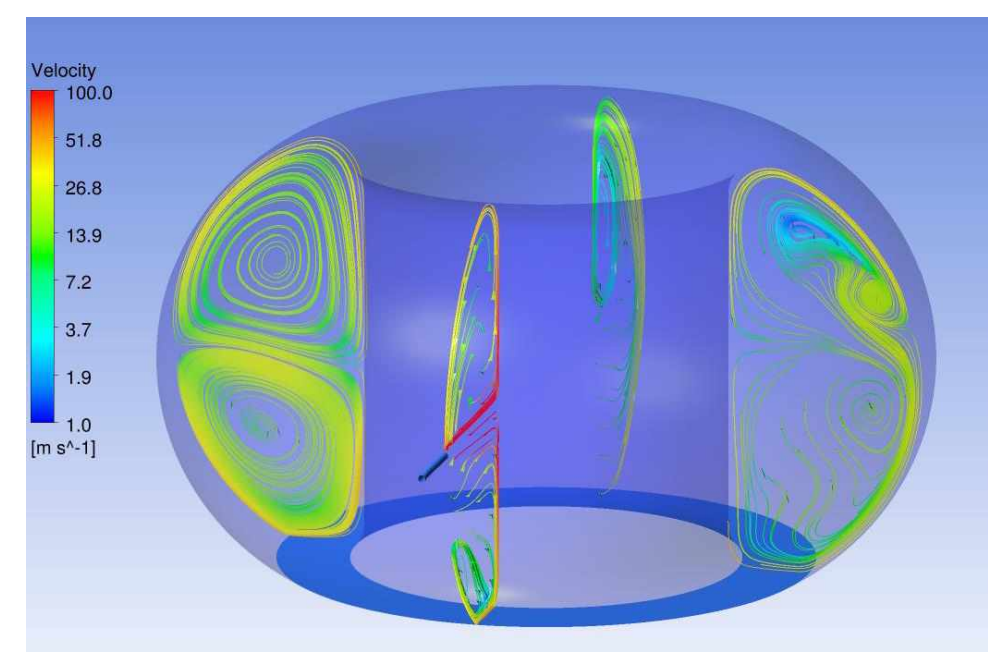

Figure 18 - airflow streamlines inside the torus $(\mathrm{t}=0.1 \mathrm{~s})$ 


\section{- Particle resuspension and dispersion}

As an illustration of the resuspension phenomenon during the calculation, Figure 19 tracks the evolution of an isosurface of the particle relative mass fraction (mass fraction divided by the maximal mass fraction) during the pressurization.

At the beginning, for the time $0.1 \mathrm{~s}$, it can be seen that the resuspension of the particles is in good agreement with the friction velocity field shown in Figure 17. Indeed, as previously mentioned, the friction velocity is the main driver of the resuspension. A particle has to overcome the adhesive forces which mainly depend on its diameter. So there is a threshold from which the resuspension occurs which explains that just a little area of the floor induces a resuspension. At the time $1 \mathrm{~s}$, the friction velocity is higher on a larger area of the floor; hence, the resuspension is more extended on this area in agreement with the friction velocity field in Figure 17. For the following times $6 \mathrm{~s}$ and $20 \mathrm{~s}$, the friction velocities on the floor are lower, so the resuspension too, and what is observed on Figure 19 is mainly the particle dispersion in the torus. The particles are carried along the wall, following the airflow streamlines as shown in Figure 18.
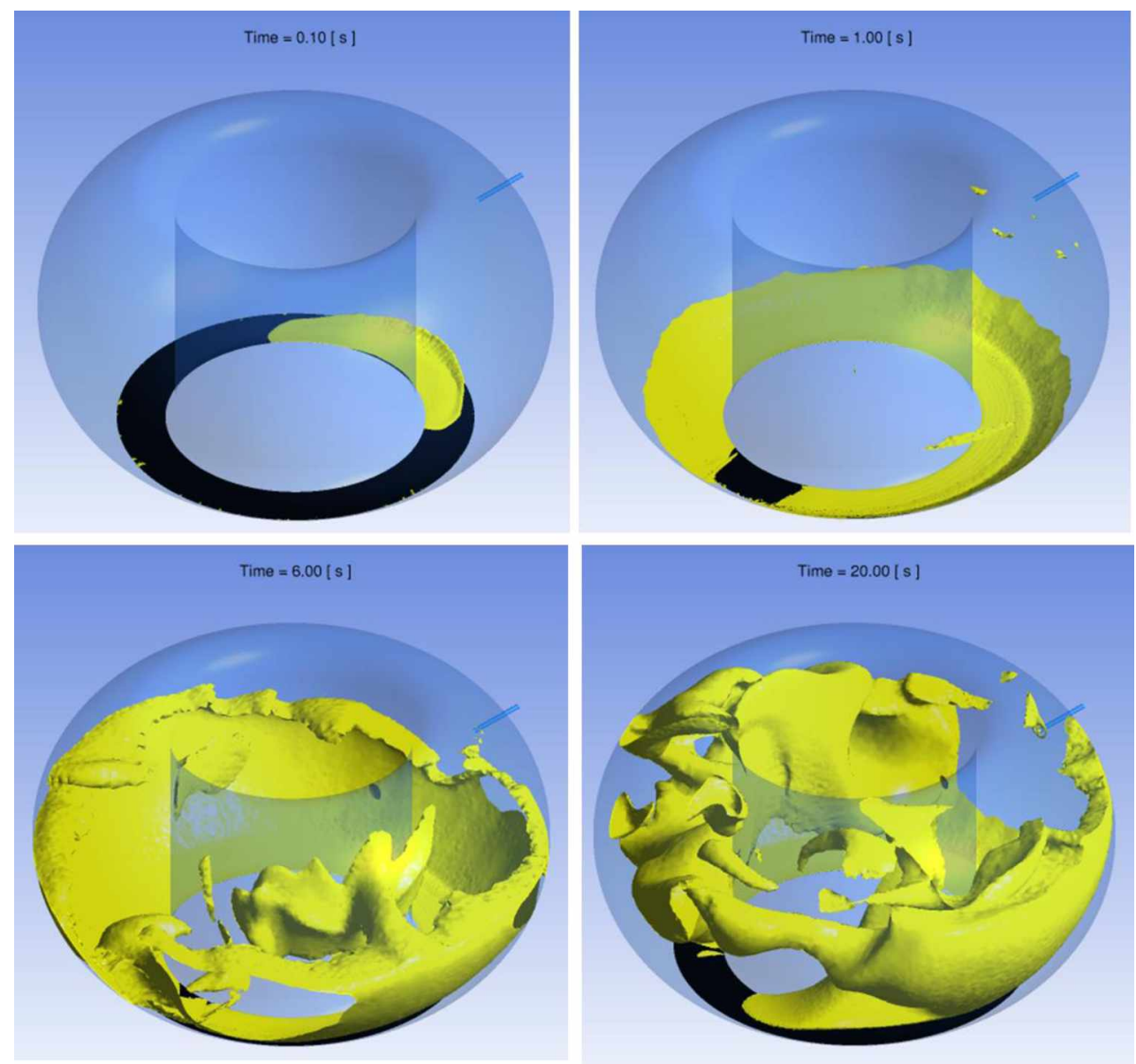

Figure 19 - time evolution of the resuspension ( $d_{p}=10$ microns) 
Figure 20 illustrates the time evolution of the resuspension rate normalized by the maximum value for each particle diameter during $20 \mathrm{~s}$, for the three studied diameters. The maximum values of the resuspension rate are indicated in bracket in the figure legend.

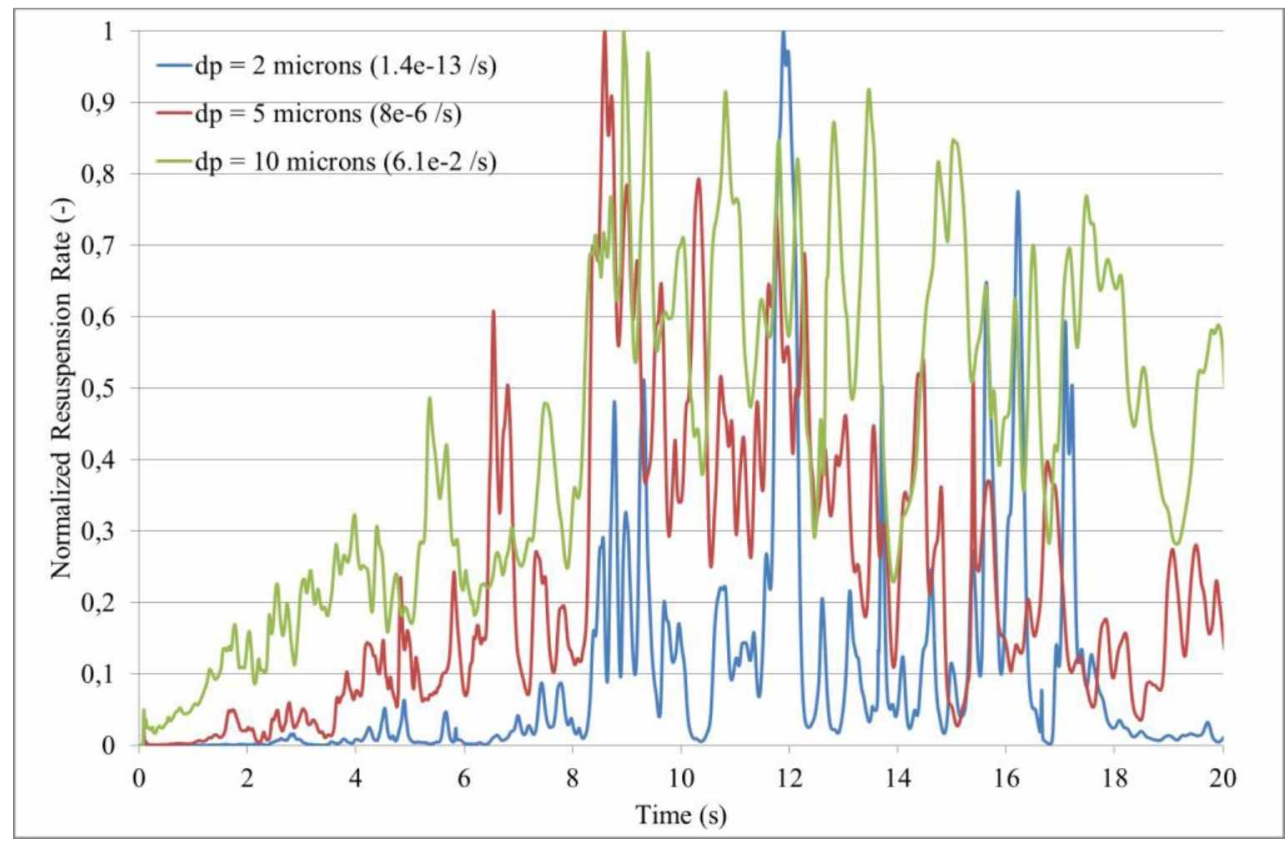

Figure 20 - resuspension rate time evolution for the three particle diameters $2 \mu \mathrm{m}, 5 \mu \mathrm{m}$ and $10 \mu \mathrm{m}$.

As tagged in the legend of the figure, the maximum resuspension rates are very different according to the diameter. Around eleven decades are observed between the resuspension rates for 2 microns aerosols and that for 10 microns.

Otherwise, the resuspension kinetics is also slightly different; for the 2 microns aerosols, the latter begin to be significantly resuspended from $8 \mathrm{~s}$, then the resuspension decreases quickly from $14 \mathrm{~s}$. In contrast, for the 5 and 10 microns aerosols, the resuspension occurs almost at the beginning of the pressurization and is always on at $20 \mathrm{~s}$.

These observations can be confirmed by the evolution of the resuspension fraction in Figure 21 . This figure presents the resuspended particle fraction (calculated by CFX with the equation (27)) in comparison with the fraction of particle that is effectively in suspension (particles that are not redeposited on the floor). Figure 22 allows to distinguish the resuspended particle fraction from the particle fraction effectively in suspension in the torus, which can be very different due to the deposition, especially for the biggest particles. 


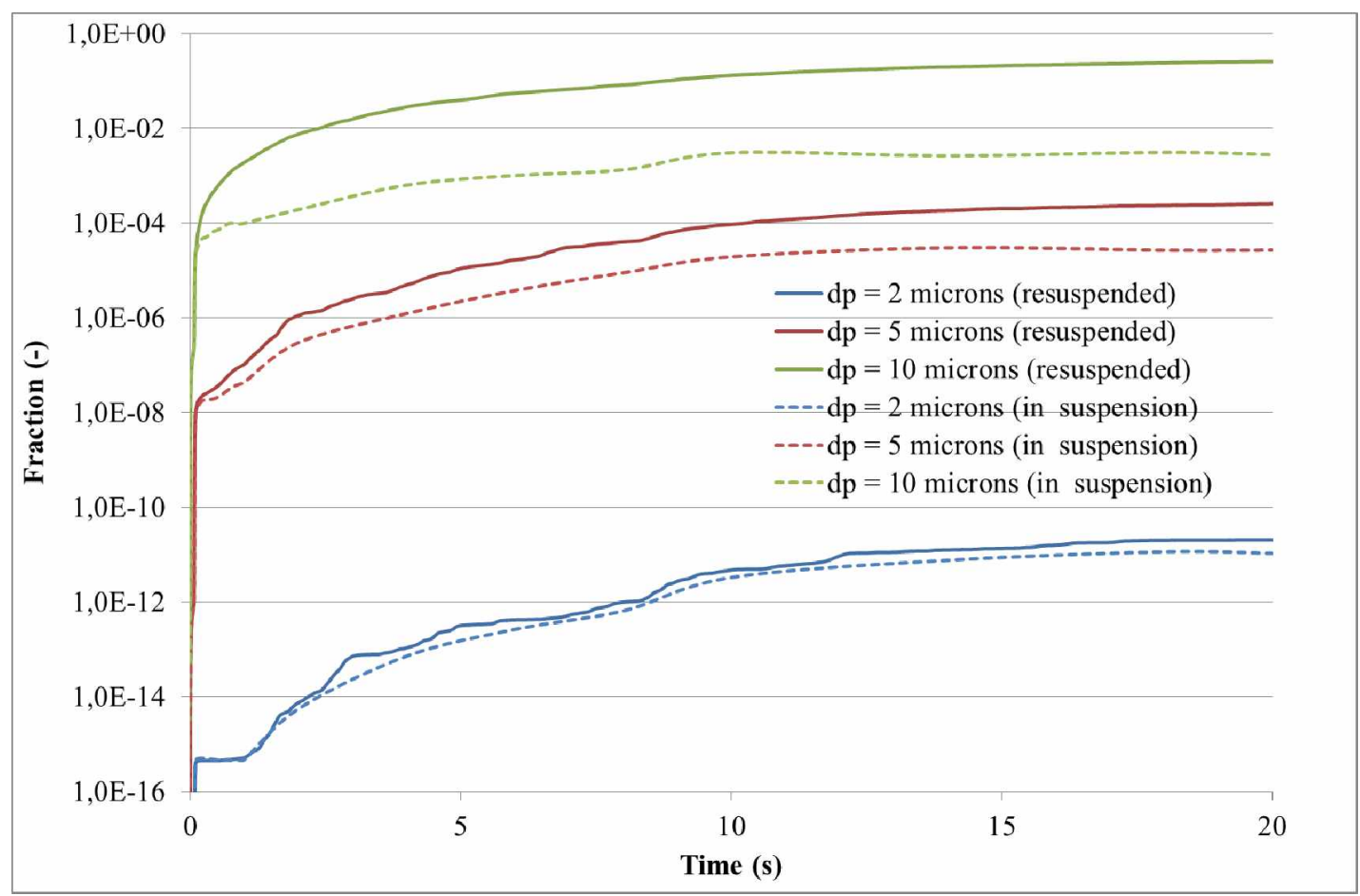

Figure 21 - resuspension and in suspension fraction

Considering the results presented in Figure 22, we propose to discuss the consequences of such resuspension fractions in terms of mass of particles and tritium one should expect in the aerosol form. Let's assume an initial deposit of $1000 \mathrm{~kg}$ of tungsten dust (safety limit for ITER is $1 \mathrm{~kg}$ of tritium and $1000 \mathrm{~kg}$ of dust according to Taylor and Cortes [30]) with diameter of $5 \mu \mathrm{m}$ in the bottom of the tokamak. After $20 \mathrm{~s}$, the fraction of particles still in suspension is below $10^{-4}$, corresponding to approximately $100 \mathrm{~g}$ of particles in suspension in the tokamak. If one considers the entire volume (i.e. $1300 \mathrm{~m}^{3}$ ), it would correspond to a concentration of $77 \mathrm{mg} / \mathrm{m}^{3}$ of $5 \mu \mathrm{m}$ tungsten particles. Explosibility of fine tungsten powders have been tested [31] and minimum values around $500 \mathrm{~g} / \mathrm{m}^{3}$ to $1 \mathrm{~kg} / \mathrm{m}^{3}$ are generally found for tungsten fine particles in the range $1 \mu \mathrm{m}$ with an ignition energy of $10 \mathrm{~kJ}$. For this particular scenario, resuspension of tungsten dust would not be safety concerns regarding potential dust explosion. On the other hand, tritium inventory is found in the biggest particles. For the same initial deposit of $1000 \mathrm{~kg}$, but in the size range $10 \mu \mathrm{m}$, approximately $1000 \mathrm{~g}$ of tungsten particles would be in suspension after $20 \mathrm{~s}$. If one assume a tritium inventory of $5 \mathrm{GBq} / \mathrm{g}$ [32], it would correspond to $5 \mathrm{TBq}$ of tritium in aerosol form. Such activity corresponds to $14 \mathrm{mg}$ of tritium trapped in the aerosol which is well below the tritium in vessel safety limit. 


\section{CONCLUSION}

This article presents the implementation of the Rock'n'Roll model in a CFD code (ANSYS CFX), its comparison with experimental tests and an application to the case of a LOVA in a toroidal geometry.

First of all, the Rock'n'Roll model integrating the adhesive force correlation of Biasi et al. [15] is described, before detailing the model implementation in ANSYS CFX, performed by the way of a source term of resuspension in the particle transport equation. The model can be used with a constant or a variable friction velocity according to the model formulation. It has to be noticed here that the Biasi correlation, which has been obtained with various experiments, could be replaced in a future work by real adhesion force distributions obtained by means of atomic force microscopy technique in the specific case of tungsten particles deposited on tungsten substrates [17]

Both formulations were first validated on experiments showing a good agreement between numerical and experimental results concerning the resuspended particle amount as well as the resuspension kinetics.

The final application of this implementation was to simulate a LOVA case in a toroidal geometry. This type of simulation is quite complex due to the lower pressure close to the vacuum which evolves with the air ingress.

These simulations carried out for 2, 5 and 10 microns aerosol sizes showed a good behaviour according to the friction velocity fields on the floor where the particles are dropped off and to the airflows which entrain the particle into the torus. The results are also in agreement with the particle resuspension physics showing a larger resuspension amount for the 10 microns particle diameter than for the 2 microns particle diameter with a difference of many decades between both.

This article demonstrates the feasibility of particle resuspension CFD simulations with the Rock'n'Roll model taking into account one layer particle surface deposition and without particleparticle interaction.

However, it can be reminded that these calculations have some limitations, particularly due to the Rock'n'Roll model use. As detailed in the article, deposited particles are assumed to be spherical, uniformly distributed on the floor, with a monolayer configuration and no interaction between particles. Moreover, this resuspension model does not take into account the Cunningham factor.

So, it appears that improvements can still be made, for example by implementing a multi-layer particle surface deposition or by using directly adhesive forces data for the studied particle-surface couples [17] 
Experimental validations of resuspension by pressurization in dedicated well-instrumented facilities such as TOSQAN facility [33] or Stardust facility [7] would be also necessary.

\section{REFERENCE}

[1] J. P. Van Dorsselaere et al., "Progress of IRSN R\&D on ITER safety assessment," J. Fusion Energy, vol. 31, no. 4, pp. 405-410, 2012.

[2] J. Roth et al., "Recent analysis of key plasma wall interactions issues for ITER," J. Nucl. Mater., vol. 390-391, pp. 1-9, Jun. 2009.

[3] L. Di Pace, E. Letellier, H. Maubert, B. Patel, and W. Raskob, "Biological hazard issues from potential releases of tritiated dust from ITER," Fusion Eng. Des., vol. 83, no. 10-12, pp. 1729-1732, Dec. 2008 .

[4] P. Cortes et al., "Optimization at the design phase of the potential impact of ITER on workers, the public and the environment," Fusion Eng. Des., vol. 85, no. 10-12, pp. $2263-$ 2267, Dec. 2010.

[5] S. Rosanvallon et al., "Dust control in tokamak environment," Fusion Eng. Des., vol. 83, no. 10-12, pp. 1701-1705, Dec. 2008.

[6] M. Glor, "Hazards due to electrostatic charging of powders," J. Electrostat., vol. 16, no. 2-3, pp. 175-191, May 1985.

[7] M. T. Porfiri, N. Forgione, S. Paci, and A. Rufoloni, "Dust mobilization experiments in the context of the fusion plants-STARDUST facility," Fusion Eng. Des., vol. 81, no. 8-14, pp. 1353-1358, Feb. 2006

[8] S. Paci, N. Forgione, F. Parozzi, and M. T. Porfiri, "Bases for dust mobilization modelling in the light of STARDUST experiments," Nucl. Eng. Des., vol. 235, no. 10-12, pp. 1129-1138, May 2005.

[9] J. . Sharpe, D. . Petti, and H.-W. Bartels, "A review of dust in fusion devices: Implications for safety and operational performance," Fusion Eng. Des., vol. 63-64, pp. 153-163, Dec. 2002

[10] J. P. Sharpe and P. W. Humrickhouse, "Dust mobilization studies in the TDMX facility," Fusion Eng. Des., vol. 81, no. 8-14, pp. 1409-1415, Feb. 2006.

[11] J. R. García-Cascales et al., "Development of a IRSN code for dust mobilisation problems in ITER," Fusion Eng. Des., 2010. 
[12] T. Gélain, A. Rondeau, S. Peillon, J. C. Sabroux, and F. Gensdarmes, "CFD modelling of the wall friction velocity field in the ITER tokamak resulting from airflow during a loss of vacuum accident-Consequences for particle resuspension," Fusion Eng. Des., vol. 100, pp. 87-99, Nov. 2015

[13] T. Honda et al., "Analyses of loss of vacuum accident (LOVA) in ITER," Fusion Eng. Des., vol. 47, no. 4, pp. 361-375, Jan. 2000.

[14] M. W. Reeks and D. Hall, "Kinetic models for particle resuspension in turbulent flows: Theory and measurement," Journal of Aerosol Science, vol. 32, no. 1. pp. 1-31, 2001.

[15] L. Biasi, A. de los Reyes, M. W. Reeks, and G. F. de Santi, "Use of a simple model for the interpretation of experimental data on particle resuspension in turbulent flows," J. Aerosol Sci., vol. 32, no. 10, pp. 1175-1200, Oct. 2001.

[16] J. N. Israelachvili, "Front Matter," Intermol. Surf. Forces, p. iii, 2011.

[17] S. Peillon et al., "Adhesion of tungsten particles on rough tungsten surfaces using Atomic Force Microscopy," J. Aerosol Sci., vol. 137, no. January, p. 105431, 2019.

[18] M. W. Reeks, J. Reed, and D. Hall, "On the resuspension of small particles by a turbulent flow," J. Phys. D. Appl. Phys., vol. 21, no. 4, pp. 574-589, 1988.

[19] K. L. Johnson, K. Kendall, and A. D. Roberts, "Surface Energy and the Contact of Elastic Solids," Proc. R. Soc. A Math. Phys. Eng. Sci., vol. 324, no. 1558, pp. 301-313, 1971.

[20] G. Ziskind, M. Fichman, and C. Gutfinger, "Resuspension of particulates from surfaces to turbulent flows-Review and analysis," J. Aerosol Sci., vol. 26, no. 4, pp. 613-644, Jun. 1995.

[21] D. Hall, "Measurements of the mean force on a particle near a boundary in turbulent flow," $J$. Fluid Mech., vol. 187, pp. 451-466, 1988.

[22] J.-I. Choi, J. R. Edwards, J. A. Rosati, and A. D. Eisner, "Large Eddy Simulation of Particle Re-suspension During a Footstep," Aerosol Sci. Technol., vol. 46, no. 7, pp. 767-780, 2012.

[23] P. Nerisson, O. Simonin, L. Ricciardi, A. Douce, and J. Fazileabasse, "Improved CFD transport and boundary conditions models for low-inertia particles," Comput. Fluids, vol. 40, no. 1, pp. 79-91, Jan. 2011.

[24] F. Gensdarmes et al., "Tore Supra carbon dust resuspension studies," Fusion Eng. Des., vol. 88, no. 9-10, pp. 2684-2687, Oct. 2013.

[25] S. Peillon, A. Roynette, C. Grisolia, and F. Gensdarmes, "Resuspension of carbon dust 
collected in Tore Supra and exposed to turbulent airflow: Controlled experiments and comparison with model," Fusion Eng. Des., vol. 89, no. 11, pp. 2789-2796, Nov. 2014.

[26] L. Biasi, A. De los Reyes, M. W. Reeks, and G. F. De Santi, "Use of a simple model for the interpretation of experimental data on particle resuspension in turbulent flows," Journal of Aerosol Science, vol. 32, no. 10. pp. 1175-1200, 2001.

[27] A. Rondeau et al., "Characterization of dust particles produced in an all-tungsten wall tokamak and potentially mobilized by airflow," J. Nucl. Mater., vol. 463, pp. 873-876, 2015.

[28] Z. Mana, "Étude sur la remise en suspension de particules suite à la marche d' un opérateur Zakaria Mana To cite this version : HAL Id : tel-01 128619," 2015.

[29] J. Xiao, J. R. Travis, W. Breitung, and T. Jordan, "Numerical analysis of hydrogen risk mitigation measures for support of ITER licensing," Fusion Eng. Des., vol. 85, no. 2, pp. 205-214, Apr. 2010.

[30] N. Taylor and P. Cortes, "Lessons learnt from ITER safety \& licensing for DEMO and future nuclear fusion facilities," Fusion Eng. Des., vol. 89, no. 9-10, pp. 1995-2000, 2014.

[31] A. Denkevits and S. Dorofeev, "Explosibility of fine graphite and tungsten dusts and their mixtures," J. Loss Prev. Process Ind., vol. 19, no. 2-3, pp. 174-180, Mar. 2006.

[32] C. Grisolia et al., "Tritium absorption and desorption in ITER relevant materials: comparative study of tungsten dust and massive samples," J. Nucl. Mater., vol. 463, pp. 885888, Aug. 2015.

[33] E. Porcheron and P. Lemaitre, "Investigation of air ingress into a vacuum vessel related to particle re-suspension and distribution for dust issues in ITER," Int. Conf. Nucl. Eng. Proceedings, ICONE, vol. 5, 2017. 